\title{
A'CAMPO CURVATURE BUMPS AND THE DIRAC PHENOMENON NEAR A SINGULAR POINT
}

\author{
SATOSHI KOIKE, TZEE-CHAR KUO AND LAURENTIU PAUNESCU
}

\begin{abstract}
The level curves of an analytic function germ can have bumps (maxima of Gaussian curvature) at unexpected points near the singularity. This phenomenon is fully explored for $f(z, w) \in \mathbb{C}\{z, w\}$, using the Newton-Puiseux infinitesimals and the notion of gradient canyon. Equally unexpected is the Dirac phenomenon: as $c \rightarrow 0$, the total Gaussian curvature of $f=c$ accumulates in the minimal gradient canyons, and nowhere else.

Our approach mimics the introduction of polar coordinates in Analytic Geometry.
\end{abstract}

\section{INTRODUCTION}

Let us first expose the idea in the real case. Consider a real analytic function germ $f(x, y)$, $f(0,0)=f_{x}(0,0)=f_{y}(0,0)=0$. The level curves $f=c, 0<|c|<\epsilon$, have "bumps" near 0 . This is illustrated in the following two examples of cusps, shown in Fig.1;

$$
f_{2}(x, y)=\frac{1}{2} x^{2}-\frac{1}{3} y^{3}, \quad f_{4}(x, y)=\frac{1}{4} x^{4}-\frac{1}{5} y^{5} .
$$

Each level curve $f_{2}=c$ attains maximum curvature (bump) when crossing the $y$-axis, along which the curvature tends to infinity as $y$ tends to zero.

A profound discovery, which one of us (Koike) learned in a lecture of A'Campo at Angers in 2000, is that bumps of such cusps can appear along peculiar arcs, not the $y$-axis.

For example, the curvature of $f_{4}=c$ is actually 0 on the $y$-axis; the maximum is attained instead as the level curve crosses $x= \pm a y^{4 / 3}+\cdots, a=(2 / 7)^{1 / 6}$. Following [10] we attribute this discovery to Nobert A'Campo, whence the name " $A$ 'Campo curvature bumps".

In this paper, we explore this phenomenon in the complex case, using the Newton-Puiseux infinitesimals (defined in [8], [9], recalled below) and the notion of "gradient canyon" (defined in \$2). (The real case, with some subtle differences, is studied in [5], where, for instance, Proposition 5.7 shows a striking difference.) Our Theorems C and D are, in a way, a continuation of Langevin [10] (total curvature), Garcia Barosso and Teissier [1] (concentration of curvature). The work of Siersma and Tibar [14] is in a different direction.

Date: November 10, 2018.

2010 Mathematics Subject Classification. Primary 14H55, Secondary 32S55.

Key words and phrases. Singular points of plane curves, Gaussian Curvature, Newton-Puiseux Infinitesimals, Dirac phenomenon. 

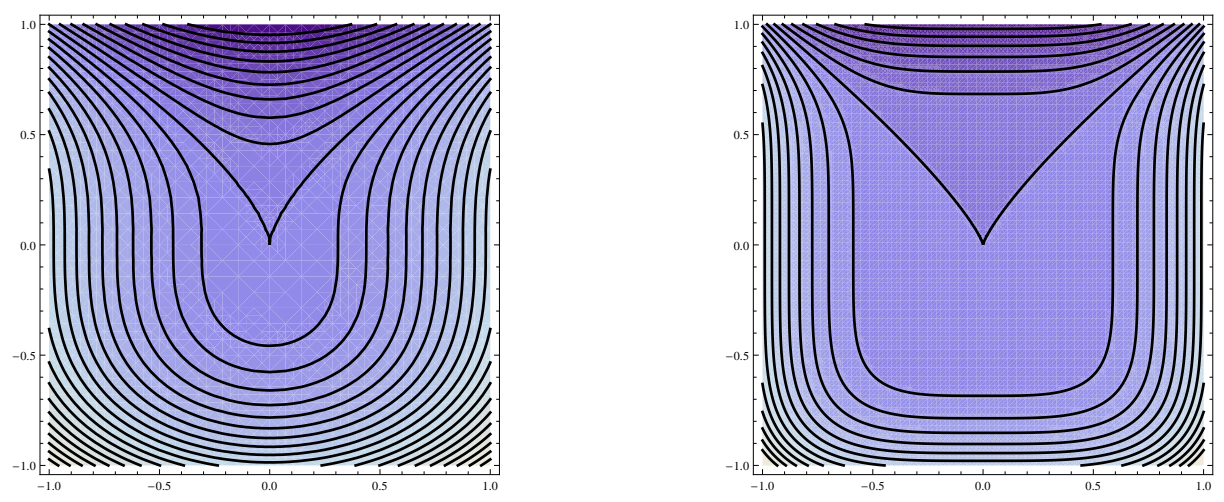

FigURE 1. $f_{2}=c, f_{4}=c$

Take $f(z, w) \in \mathbb{C}\{z, w\}$. A level curve $\mathcal{S}_{c}: f(z, w)=c$ is a Riemann Surface in $\mathbb{C}^{2}$, having Gaussian curvature (at regular points)

$$
K(z, w)=\frac{2\left|\Delta_{f}(z, w)\right|^{2}}{\left(\left|f_{z}\right|^{2}+\left|f_{w}\right|^{2}\right)^{3}}, \quad \Delta_{f}(z, w):=\left|\begin{array}{ccc}
f_{z z} & f_{z w} & f_{z} \\
f_{w z} & f_{w w} & f_{w} \\
f_{z} & f_{w} & 0
\end{array}\right| .
$$

This is actually the negative of the usual Gaussian curvature defined in text books (2]). The formula is due to Ness ([12]); for convenience we give a proof in $₫ 8$ (I).

Consider a holomorphic map germ with parameter $t$,

$$
\alpha:(\mathbb{C}, 0) \longrightarrow\left(\mathbb{C}^{2}, 0\right), \quad \alpha(t)=(z(t), w(t)) \not \equiv 0 .
$$

The image set germ $\alpha_{*}:=\operatorname{Im}(\alpha)$ is an irreducible curve germ at 0 in $\mathbb{C}^{2}$, also called a holomorphic arc at 0 . It has a unique (complex) tangent line $T\left(\alpha_{*}\right)$ at $0, T\left(\alpha_{*}\right) \in \mathbb{C} P^{1}$.

We call $\alpha_{*}$ a Newton-Puiseux infinitesimal at $T\left(\alpha_{*}\right)$.

The Enriched Riemann Sphere ("enriched" with infinitesimals) is, by definition,

$$
\mathbb{C} P_{\text {enriched }}^{1}:=\left\{\alpha_{*}\right\} \text {, the set of holomorphic arcs at } 0 \in \mathbb{C}^{2},
$$

furnished with a number of structures defined in $\$ 2$,

The image of $t \mapsto(a t, b t)$ is identified with $[a: b] \in \mathbb{C} P^{1}$, so that $\mathbb{C} P^{1} \subset \mathbb{C} P_{\text {enriched }}^{1}$.

Let $f(z, w) \in \mathbb{C}\{z, w\}$ be given. Take $\alpha_{*}$, and a parametrization $\alpha(t)$.

Suppose $K(\alpha(t)) \not \equiv 0$. Then there exists a unique pair $(a, L)$ such that

$$
\lim _{t \rightarrow 0} \frac{K(\alpha(t))}{\|\alpha(t)\|^{L}}=a, \quad a>0, L \in \mathbb{Q} \text { (rationals), }
$$

where $(a, L)$ depends on $\alpha_{*}$, but not on the choice of $\alpha(t)$. This is shown in $\oint 8$ (II).

We can write (1.3) as

$$
K(\alpha(t)) \sim a\|\alpha(t)\|^{L}
$$


where $A(t) \sim B(t)$ means $\lim _{t \rightarrow 0} A(t) / B(t)=1$. Accordingly, we define the "value space"

$$
\mathcal{V}:=\left\{a \delta^{L} \mid a \neq 0, L \in \mathbb{Q}\right\} \cup\left\{O_{\mathcal{V}}\right\},
$$

where $\delta$ is a symbol,

$$
a \delta^{L}:=(a, L), \quad O_{\mathcal{V}}:=0 \delta^{\infty}:=(0, \infty) ;
$$

the latter is added so that when $K \equiv 0$ along $\alpha_{*}$, the value is $O_{\mathcal{V}}$, the "origin" of $\mathcal{V}$.

A lexicographic ordering on $\mathcal{V}$ is defined: $O_{\nu}$ is the smallest element,

$$
a \delta^{L}>a^{\prime} \delta^{L^{\prime}} \text { if and only if either } L<L^{\prime} \text {, or else } L=L^{\prime}, a>a^{\prime} .
$$

We also define $a \delta^{L} \gg a^{\prime} \delta^{L^{\prime}}$ (substantially larger than) if $L<L^{\prime}$. Thus, for example,

$$
2 \delta^{3 / 2}>\delta^{3 / 2} \gg 10^{10} \delta^{2} \gg O_{\mathcal{V}}
$$

Let $f(z, w)$ be given. Using (1.3) , we define the curvature function

$$
K_{*}: \mathbb{C} P_{\text {enriched }}^{1} \longrightarrow \mathcal{V}, \quad \alpha_{*} \mapsto a \delta^{L},
$$

and also the exponent function

$$
L_{*}: \mathbb{C} P_{\text {enriched }}^{1} \longrightarrow \mathbb{Q} \cup\{\infty\}, \quad \alpha_{*} \mapsto L .
$$

What we do in this paper mimics the shifting from Cartesian coordinates to the polar coordinates in Analytic Geometry, where, for example, in order to study

$$
\left.x^{2}-y^{2}=\left(x^{2}+y^{2}\right)^{2}, \quad(x, y) \in \mathbb{R}^{2}, \quad \text { (the "figure } \infty \text { " curve, }\right)
$$

we "lift" it to $r^{2}=\cos 2 \theta$ in the $(r, \theta)$-space.

We proceed similarly, in order to understand the behaviour of $K(z, w)$ as $(z, w) \rightarrow 0$ in $\mathbb{C}^{2}$, we study the function $K_{*}$ on $\mathbb{C} P_{\text {enriched }}^{1}$. This turns out to be quite effective and convenient, even though $\mathbb{C} P_{\text {enriched }}^{1}$, being a space of arcs, is infinite dimensional.

In §2, the main concepts, the A'Campo bump, the gradient canyon, the infinitesimal disc and line, etc., are introduced, and the main results, Theorem A to Theorem E, are stated.

In Theorem A, supplemented by Theorem B, we show how to compute the A'Campo bumps. Examples are given to expose the arcs in $\mathbb{C}^{2}$ along which the bumps appear.

We then study the total Gaussian curvature. In Theorem C, the integral of the curvature over a gradient canyon, as $c \rightarrow 0$, is given by a Gauss-Bonnet type formula.

Theorem D asserts that the curvature on an infinitesimal line concentrates in the gradient canyons contained therein, and nowhere else; in other words, the integral of the curvature, as $c \rightarrow 0$, behaves like that of a Dirac function. This is a much more precise information than that given in [10], [1].

The integral extends to a measure on $\mathbb{C}_{\text {enriched }}$, the $\sigma$-algebra of measurable sets being generated by the enriched discs (Remark 2.10, Remark6.9).

In Theorem E the polars are perturbed within the canyons to create twin networks of iterated torus knots; the Milnor number $\mu_{f}$ is expressed as their linking number. 
Throughout this paper we assume $f(0,0)=0$ and $f(z, w)$ is not of the form

$$
f(z, w)=\text { unit } \cdot[z-\zeta(w)]^{m} \text {, }
$$

where $\zeta(w)$ is an integral power series. Indeed, if (1.7) holds, then $f(z, w)^{1 / m}$ is holomorphic and non-singular, having the same Gaussian curvature $K(z, w)$ as $f(z, w)$.

We do not assume 0 is an isolated singularity of $f(z, w)$.

Theorems A, B, C have been announced in [4].

We are grateful to the referees, their constructive criticism has led to a much better presentation of this paper. We also thank Don Cartwright for his advice on Carathéodory's Extension Theorem.

\section{The Space $\mathbb{C} P_{\text {enriched }}^{1}$ And the Main Theorems}

The classical Newton-Puiseux Theorem asserts that the field $\mathbb{F}$ of convergent fractional power series in an indeterminate $y$ is algebraically closed. ([16], [17].)

Recall that a non-zero element of $\mathbb{F}$ is a (finite or infinite) convergent series

$$
\alpha(y)=a_{0} y^{n_{0} / N}+\cdots+a_{i} y^{n_{i} / N}+\cdots, \quad n_{0}<n_{1}<\cdots,
$$

where $0 \neq a_{i} \in \mathbb{C}, n_{i} \in \mathbb{Z}$ (integers), $N \in \mathbb{Z}^{+}$(positive integers), and

$$
G C D\left(N, n_{0}, n_{1}, \ldots\right)=1, \quad \lim \sup \left|a_{k}\right|^{\frac{1}{n_{k}}}<\infty .
$$

We shall also write $\alpha(y)$ as $\alpha$. The conjugates of $\alpha$ are

$$
\alpha_{c o n j}^{(k)}(y):=\sum a_{i} \theta^{k n_{i}} y^{n_{i} / N}, \quad 0 \leq k \leq N-1, \quad \theta:=e^{\frac{2 \pi \sqrt{-1}}{N}} .
$$

The order of $\alpha$ is

$$
O(\alpha):=O_{y}(\alpha):=\frac{n_{0}}{N} \text { if } \alpha \neq 0 ; \quad O(\alpha):=\infty \text { if } \alpha=0 .
$$

The Puiseux multiplicity of $\alpha$ is, by definition,

$$
m_{\text {puiseux }}(\alpha):=N \text {. }
$$

Let $e \in \mathbb{Q}$, or $e=\infty$, be given. The $e$-jet of $\alpha$ is, by definition,

$$
J^{(e)}(\alpha)(y):= \begin{cases}\alpha(y) \text { with all terms } y^{q}, q>e, \text { deleted, } & \text { if } e<\infty \\ \alpha(y) & \text { if } e=\infty\end{cases}
$$

Example 2.1. The equation $z^{2}-y^{3}=0$ has a pair of conjugate roots $\pm y^{3 / 2}$,

$$
\theta=-1, \quad O_{y}\left( \pm y^{3 / 2}\right)=3 / 2, \quad m_{\text {puiseux }}\left( \pm y^{3 / 2}\right)=2 .
$$

Next, consider the following polynomial equation of degree 4 over $\mathbb{F}$ :

$$
\left(z^{2}-y^{3}\right)^{2}-z y^{5}=0 \text {. }
$$

Using the Newton Polygon method ([16]), the roots are found to be

$$
y^{\frac{3}{2}} \pm \frac{1}{2} y^{\frac{7}{4}}+\cdots,-y^{\frac{3}{2}} \pm \frac{\sqrt{-1}}{2} y^{\frac{7}{4}}+\cdots
$$


where all four are conjugates, $\theta=\sqrt{-1}$. Let $\alpha$ denote any one of them, then

$$
m_{\text {puiseux }}(\alpha)=4, O_{y}(\alpha)=\frac{3}{2}, \quad J^{(3 / 2)}(\alpha)= \pm y^{\frac{3}{2}}, m_{\text {puiseux }}\left(J^{(3 / 2)}(\alpha)\right)=2 .
$$

Now, $\mathbb{C} P_{\text {enriched }}^{1}$ is the union of two "enriched complex lines"

$$
\mathbb{C} P_{\text {enriched }}^{1}=\mathbb{C}_{\text {enriched }} \cup \mathbb{C}_{\text {enriched }}^{\prime}
$$

as in Projective Geometry, where

$$
\mathbb{C}_{\text {enriched }}:=\left\{\beta_{*} \mid T\left(\beta_{*}\right) \neq[1: 0]\right\}, \quad \mathbb{C}_{\text {enriched }}^{\prime}:=\left\{\beta_{*} \mid T\left(\beta_{*}\right) \neq[0: 1]\right\} .
$$

Let us introduce coordinates on $\mathbb{C}_{\text {enriched }}$. Consider

$$
\mathbb{F}_{0}:=\left\{\alpha \in \mathbb{F} \mid O_{y}(\alpha) \geq 0\right\}, \mathbb{F}_{1}:=\left\{\alpha \mid O_{y}(\alpha) \geq 1\right\}, \mathbb{F}_{1^{+}}:=\left\{\alpha \mid O_{y}(\alpha)>1\right\},
$$

where $\mathbb{F}_{0}$ is an integral domain with quotient field $\mathbb{F} ; \mathbb{F}_{1}, F_{1^{+}}$are ideals of $\mathbb{F}_{0}$.

Take any $\alpha \in \mathbb{F}_{1}$ in (2.3). The map germ

$$
\alpha_{\text {para }}:(\mathbb{C}, 0) \longrightarrow\left(\mathbb{C}^{2}, 0\right), \quad t \mapsto\left(\alpha\left(t^{N}\right), t^{N}\right), \quad N:=m_{\text {puiseux }}(\alpha),
$$

is holomorphic. Hence, as in (1.2), the holomorphic arc $\alpha_{*} \in \mathbb{C}_{\text {enriched }}$ is defined.

If $N$ is replaced by $k N, k \in \mathbb{Z}^{+}, \alpha_{*}$, being a set germ, would not change.

Of course, all conjugates of $\alpha$ lead to the same holomorphic arc $\alpha_{*}$. For example, the roots in (2.2) give rise to the holomorphic arc with parametrisation $\left(t^{6}+\frac{1}{2} t^{7}+\cdots, t^{4}\right)$.

The Newton-Puiseux coordinate system on $\mathbb{C}_{\text {enriched }}$ is, by definition, the surjection

$$
\pi: \mathbb{F}_{1} \longrightarrow \mathbb{C}_{\text {enriched }}, \quad \alpha \mapsto \alpha_{*} .
$$

When $\alpha_{*}$ is given, the conjugate class of $\alpha$ is unique. That is, $\pi$ induces a bijection between the set of conjugate classes in $\mathbb{F}_{1}$ and $\mathbb{C}_{\text {enriched }}$.

Definition 2.2. Let $\alpha_{*} \in \mathbb{C}_{\text {enriched }}$ be given. Take $e, 1 \leq e<\infty$, and $\rho \geq 0$. Let

$$
\mathcal{D}^{(e)}\left(\alpha_{*} ; \rho\right):=\left\{\beta_{*}\left|\beta(y)=\left[J^{(e)}(\alpha)(y)+c y^{e}\right]+\cdots,\right| c \mid \leq \rho\right\},
$$

where "..." means "higher order terms", and

$$
\begin{aligned}
\mathcal{L}^{(e)}\left(\alpha_{*}\right): & =\mathcal{D}^{(e)}\left(\alpha_{*} ; \infty\right):=\cup_{0<\rho<\infty} \mathcal{D}^{(e)}\left(\alpha_{*} ; \rho\right) \\
& =\left\{\beta_{*}\left|\beta(y)=\left[J^{(e)}(\alpha)(y)+c y^{e}\right]+\cdots,\right| c \mid<\infty\right\} .
\end{aligned}
$$

In particular,

$$
\mathcal{L}^{(1)}\left(\alpha_{*}\right)=\mathbb{C}_{\text {enriched }}, \quad \mathcal{D}^{(e)}\left(\alpha_{*} ; 0\right):=\left\{\beta_{*} \mid \beta(y)=J^{(e)}(\alpha)(y)+\cdots\right\} .
$$

We call $\mathcal{D}^{(e)}\left(\alpha_{*}, \rho\right)$ the enriched (complex) disc along $\alpha_{*}$, of order $e$, radius $\rho$, and $\mathcal{L}^{(e)}\left(\alpha_{*}\right)$ the enriched line along $\alpha_{*}$ of order e.

When e $>1$, we also call $\mathcal{D}^{(e)}$ an infinitesimal disc, and $\mathcal{L}^{(e)}$ an infinitesimal line.

For convenience, we define

$$
\mathcal{D}^{(\infty)}\left(\alpha_{*}\right):=\mathcal{L}^{(\infty)}\left(\alpha_{*}\right):=\left\{\alpha_{*}\right\}, \text { a singleton. }
$$


Finally, for $1 \leq e \leq \infty$, using (2.1), we define the e-jet map:

$$
J^{(e)}: \mathbb{C}_{\text {enriched }} \rightarrow \mathbb{C}_{\text {enriched }}, \quad \alpha_{*} \mapsto J^{(e)}\left(\alpha_{*}\right):=J^{(e)}(\alpha)_{*}
$$

When $\alpha_{*}$ is given, the right-hand side of (2.6) does not depend on the choices of $\alpha$ in the conjugate class for $\alpha_{*}$. We can use any $\alpha$ in the conjugate class. Note also that

$$
J^{(e)}\left(\alpha_{*}\right)=J^{(e)}\left(\tilde{\alpha}_{*}\right) \Longleftrightarrow \mathcal{D}^{(e)}\left(\alpha_{*} ; \rho\right)=\mathcal{D}^{(e)}\left(\tilde{\alpha}_{*} ; \rho\right),
$$

and that

$$
O_{y}(\alpha-\tilde{\alpha}) \geq e \Longleftrightarrow \mathcal{L}^{(e)}\left(\alpha_{*}\right)=\mathcal{L}^{(e)}\left(\tilde{\alpha}_{*}\right)
$$

Two enriched lines are either disjoint, or else one contains the other (or are equal),

$$
\mathcal{L}^{(e)}\left(\alpha_{*}\right) \subsetneq \mathcal{L}^{(\tilde{e})}\left(\tilde{\alpha}_{*}\right) \Longleftrightarrow e>\tilde{e} \text { and } O_{y}(\alpha-\tilde{\alpha}) \geq \tilde{e}
$$

Let $f(z, w)$ be given. For convenience, let us apply a generic unitary transformation, if necessary, to bring $f(z, w)$ to the form

$$
f(z, w):=H_{m}(z, w)+H_{m+1}(z, w)+\cdots, H_{m}(1,0) \neq 0, O(f)=m,
$$

where $H_{k}$ is a homogeneous $k$-form. (When $H_{m}(1,0) \neq 0$, we say $f$ is mini-regular in $z$.)

A unitary transformation preserves the metric, hence also the Gaussian curvature.

From now on we shall restrict our attention to $\mathbb{C}_{\text {enriched }}$.

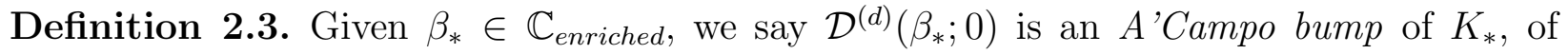
degree $d, 1 \leq d<\infty$, if the following three conditions are satisfied.

(1) The curvature function $K_{*}$ is constant on $\mathcal{D}^{(d)}\left(\beta_{*} ; 0\right)$. That is,

$$
J^{(d)}\left(\xi_{*}\right)=J^{(d)}\left(\beta_{*}\right) \Longrightarrow K_{*}\left(\xi_{*}\right)=K_{*}\left(\beta_{*}\right) ;
$$

(2) If $\rho>0$ is sufficiently small, then

$$
J^{(d)}\left(\xi_{*}\right) \in \mathcal{D}^{(d)}\left(\beta_{*} ; \rho\right) \Longrightarrow K_{*}\left(\xi_{*}\right) \leq K_{*}\left(\beta_{*}\right) ;
$$

where " $<$ " holds for generic $\xi_{*}$, i.e., if $a$ is generic, $|a| \leq \rho$, then

$$
\xi(y)=\left[J^{(d)}(\beta)(y)+a y^{d}\right]+\cdots \Longrightarrow K_{*}\left(\xi_{*}\right)<K_{*}\left(\beta_{*}\right) ;
$$

(3) In the case $d>1$, there exists $\epsilon>0$ such that

$$
J^{(d-\epsilon)}\left(\xi_{*}\right)=J^{(d-\epsilon)}\left(\beta_{*}\right), J^{(d)}\left(\xi_{*}\right) \neq J^{(d)}\left(\beta_{*}\right) \Longrightarrow K_{*}\left(\xi_{*}\right) \ll K_{*}\left(\beta_{*}\right) .
$$

Now, let $f(z, w)$ be as in (2.9) . Consider the Newton-Puiseux factorizations:

$$
f(z, y)=\text { unit } \cdot \prod_{i=1}^{m}\left(z-\zeta_{i}(y)\right), \quad f_{z}(z, y)=\text { unit } \cdot \prod_{j=1}^{m-1}\left(z-\gamma_{j}(y)\right)
$$

where $\zeta_{i}, \gamma_{j} \in \mathbb{F}_{1}$. Each $\gamma_{j}$, and $\gamma_{j *}$, is called a polar.

If a polar $\gamma$ is also a root of $f$, i.e. $f(\gamma(y), y) \equiv 0$, then it is a multiple root of $f$. We shall see in Theorem A that such polars do not give rise to A'Campo bumps. 
If there are $k$ distinct $\zeta_{i}$ in (2.10), there are exactly $k-1$ polars (counting multiplicities) which are not roots of $f$. This is a consequence of Theorem 2.1 in [7] (or Lemma 3.3 in [6]). Since $f$ is not of the form (1.7), we have $k \geq 2$. Hence at least one such polar exists.

The following implication follows from the Chain Rule in Calculus: For any $\alpha \in \mathbb{F}_{1}$,

$$
f_{z}(\alpha(y), y) \equiv f_{w}(\alpha(y), y) \equiv 0 \Longrightarrow f(\alpha(y), y) \equiv 0
$$

Now take a polar $\gamma, f(\gamma(y), y) \not \equiv 0$. By (2.11), $\gamma$ is not a common Newton-Puiseux root of $f_{z}, f_{w}$. Hence, if $q$ is sufficiently large, then

$$
O_{y}(\|\operatorname{Grad} f(\gamma(y), y)\|)=O_{y}\left(\left\|\operatorname{Grad} f\left(\gamma(y)+u y^{q}, y\right)\right\|\right), \forall u \in \mathbb{C} .
$$

Let $d_{g r}(\gamma)$ denote the smallest number $q$ such that (2.12) holds for generic $u \in \mathbb{C}$.

In the case $f(\gamma(y), y) \equiv 0$, write $d_{g r}(\gamma):=\infty$.

Definition 2.4. Take a polar $\gamma, d:=d_{g r}(\gamma)$. The gradient canyon of $\gamma_{*}$ is, by definition,

$$
\mathcal{G C}\left(\gamma_{*}\right):=\mathcal{L}^{(d)}\left(\gamma_{*}\right), \quad 1 \leq d \leq \infty
$$

We call $d_{g r}\left(\gamma_{*}\right):=d_{g r}(\gamma)$ the gradient degree of $\gamma$, and the degree of $\mathcal{G C}\left(\gamma_{*}\right)$.

We say $\mathcal{G C}\left(\gamma_{*}\right)$ is minimal if $d_{g r}\left(\gamma_{*}\right)<\infty$ and for every polar $\gamma_{j}$ with $d_{g r}\left(\gamma_{j}\right)<\infty$,

$$
\mathcal{G C}\left(\gamma_{j *}\right) \subseteq \mathcal{G C}\left(\gamma_{*}\right) \Longrightarrow \mathcal{G C}\left(\gamma_{j *}\right)=\mathcal{G C}\left(\gamma_{*}\right)
$$

(The gradient canyons are not topological invariants, see §8(III).)

Example 2.5. For $f=z^{m}-w^{n}, 2 \leq m \leq n, \gamma=0$ is the only polar,

$$
\mathcal{G C}\left(0_{*}\right)=\left\{\alpha_{*} \mid \alpha(y)=u y^{d_{g r}(\gamma)}+\cdots, u \in \mathbb{C}\right\}, \quad d_{g r}(\gamma)=\frac{n-1}{m-1} .
$$

Example 2.6. Take $f=z^{4}-2 z^{2} w^{2}-w^{100}$, having polars $\gamma_{1}=0, \gamma_{2}, \gamma_{3}= \pm w$. We find

$$
d_{g r}\left(\gamma_{1}\right)=97, \quad d_{g r}\left(\gamma_{2}\right)=d_{g r}\left(\gamma_{3}\right)=1 .
$$

Here $\mathcal{G C}\left(\gamma_{1 *}\right)$ is minimal, but $\mathcal{G C}\left(\gamma_{2 *}\right)=\mathcal{G C}\left(\gamma_{3 *}\right)=\mathbb{C}_{\text {enriched }}$ is not.

Next consider $g=z^{4}-2 z^{2} w^{2}$, with $\gamma_{1}=0, \gamma_{2}, \gamma_{3}= \pm w$. Then

$$
\mathcal{G C}\left(\gamma_{1 *}\right)=\left\{\gamma_{1 *}\right\} \subset \mathcal{G C}\left(\gamma_{2 *}\right)=\mathcal{G C}\left(\gamma_{3 *}\right)=\mathbb{C}_{\text {enriched }}
$$

The latter is minimal, containing the former, $d_{g r}\left(\gamma_{1 *}\right)=\infty$.

We shall see in Theorem $B$ that $\mathbb{C}_{\text {enriched }}$ is a minimal canyon only in very exceptional cases, and that no minimal canyon, except $\mathbb{C}_{\text {enriched }}$, can contain a singleton canyon.

Now take a polar $\gamma, d_{g r}(\gamma)<\infty$. Define $L_{\gamma} \in \mathbb{Q}$, and $R_{\gamma}: \mathbb{C} \rightarrow \mathbb{C}$, as follows.

First, we apply a unitary transformation, if necessary, so that $T\left(\gamma_{*}\right)=[0: 1], \gamma \in \mathbb{F}_{1}$.

For brevity let us write $d:=d_{g r}(\gamma)$. If $d>1$, define $L_{\gamma}$ and $R_{\gamma}(u)$ by the equation

$$
K\left(\gamma(y)+u y^{d}, y\right)=2 R_{\gamma}(u) y^{2 L_{\gamma}}+\cdots, \quad R_{\gamma}(u) \not \equiv 0 .
$$

Note that for generic $u \in \mathbb{C}$,

$$
L_{\gamma}=\frac{1}{2} L_{*}\left(\left(\gamma+u y^{d}\right)_{*}\right), \quad K_{*}\left(\left(\gamma+u y^{d}\right)_{*}\right)=\left(2 R_{\gamma}(u), L_{*}(\gamma)\right),
$$


where $L_{*}$ was defined in (1.6).

In the case $d=1$, we define $L_{\gamma}$ and $R_{\gamma}(u)$ by

$$
K\left(\gamma(y)+\frac{u y}{\sqrt{1+|u|^{2}}}, \frac{y}{\sqrt{1+|u|^{2}}}\right)=2 R_{\gamma}(u) y^{2 L_{\gamma}}+\cdots, \quad R_{\gamma}(u) \not \equiv 0 .
$$

Since $\gamma(y)$ has no linear term, (2.14) remains true.

(We can use (2.15) also for the case $d>1$; but (2.13) is easier for computation.)

Lemma 2.7. The function $R_{\gamma}(u)(\not \equiv 0)$ is defined and continuous for all $u \in \mathbb{C}$,

$$
R_{\gamma}(u) \geq 0, \lim _{u \rightarrow \infty} R_{\gamma}(u)=0 ; \quad L_{\gamma}=-d_{g r}(\gamma) .
$$

In particular, $R_{\gamma}(u)$ is bounded, hence has at least one local maximum.

The absolute maximum is of course attained. This lemma will be proved in $\$ 5$.

Theorem A. Take a minimal gradient canyon $\mathcal{G C}\left(\gamma_{*}\right)$. Take $c \in \mathbb{C}$ such that $R_{\gamma}(c)$ is a local maximum of $R_{\gamma}(u)$ and let

$$
\beta(y):=\left[J^{(d)}(\gamma)(y)+c y^{d}\right]+\cdots, \quad d:=d_{g r}\left(\gamma_{*}\right) .
$$

Then $\mathcal{D}^{(d)}\left(\beta_{*} ; 0\right)$ is an A'Campo bump. All A'Campo bumps are of this form.

Since $f(z, w)$ is not of the form (1.7),$d_{g r}(\gamma)<\infty$ for at least one polar $\gamma$. There exists at least one minimal gradient canyon, hence at least one A'Campo bump.

Example. For $f_{2}(z, w)=\frac{1}{2} z^{2}-\frac{1}{3} w^{3}$, there is only one polar $\gamma=0$, having $d_{g r}(\gamma)=2$,

$$
R_{\gamma}(u)=\left(|u|^{2}+1\right)^{-3}, \quad L_{\gamma}=-2, K\left(\gamma+u y^{2}, y\right)=2 R_{\gamma}(u) y^{-4}+\cdots .
$$

Hence $R_{\gamma}(u)$ is maximum at $u=0$. The A'Campo bump is $\mathcal{D}^{(2)}\left(0_{*} ; 0\right)$.

Next, consider $f_{4}(z, w)=\frac{1}{4} z^{4}-\frac{1}{5} w^{5}$, having only one polar $\gamma=0$,

$$
d:=d_{g r}(\gamma)=\frac{4}{3}, \Delta=z^{2} w^{3}\left(4 z^{4}-3 w^{5}\right), R_{\gamma}(u)=\frac{9|u|^{4}}{\left(|u|^{6}+1\right)^{3}}, L_{\gamma}=-d .
$$

Here $R_{\gamma}(u)$ is maximum on the circle $|u|=(2 / 7)^{1 / 6}$, like a volcanic ring; each $u$ on the ring leads to an A'Campo bump. This infinite family of bumps shows up in Fig.1 as two real arcs. (However, $R_{\gamma}(0)=0, \mathcal{D}^{(e)}\left(0_{*} ; 0\right)$ is not a bump; in Fig. 1, $K=0$ along the $y$-axis.)

Now let us factor the initial form $H_{m}(z, w)$ in (2.9):

$$
H_{m}(z, w)=c\left(z-z_{1} w\right)^{m_{1}} \cdots\left(z-z_{r} w\right)^{m_{r}}, m_{i} \geq 1, z_{i} \neq z_{j} \text { if } i \neq j,
$$

and $1 \leq r \leq m, m=m_{1}+\cdots+m_{r}, c \neq 0$.

If $r=m$, all $m_{i}=1$. If $r<m$ then $H_{m}(z, w)$ is degenerate, and vice versa.

Theorem B. Every gradient canyon of degree $d, 1<d<\infty$, is minimal; gradient canyons with $1<d_{g r} \leq \infty$ (singleton canyons included) are mutually disjoint.

There are exactly $r-1$ polars of gradient degree 1 (counting multiplicities), they all have $\mathbb{C}_{\text {enriched }}$ as gradient canyon. (If $r=1$, every gradient canyon of degree $<\infty$ is minimal.)

In the case $1<r \leq m, \mathbb{C}_{\text {enriched }}$ is minimal if and only if $f(z, w)$ has exactly $r$ distinct roots $\zeta_{i}$ in (2.10). (In particular, if $H_{m}(z, w)$ is non-degenerate, $\mathbb{C}_{\text {enriched }}$ is minimal.) 
Theorems A and B will be proved in \$5. They are used to find all A'Campo bumps.

Now we state Theorem $\mathrm{C}$ and Theorem D, to be proved in $\S 6$.

Definition 2.8. Consider a given enriched disc of finite order and positive radius

$$
\mathcal{D}^{(e)}\left(\alpha_{*} ; \rho\right), \quad 1 \leq e<\infty, 0<\rho<\infty .
$$

Take a compact disc in $\mathbb{C}^{2}$ :

$$
D_{i s c}(0 ; \eta):=\left\{(z, w) \in \mathbb{C}^{2} \mid \sqrt{|z|^{2}+|w|^{2}} \leq \eta\right\}, \eta>0 \text { sufficiently small. }
$$

The horn domain (in $\mathbb{C}^{2}$ ) associated to $\mathcal{D}^{(e)}\left(\alpha_{*} ; \rho\right)$ is the compact subset of $\mathbb{C}^{2}$ :

$$
H_{\text {orn }}^{(e)}\left(\alpha_{*} ; \rho ; \eta\right):=\left\{(z, w) \in D_{i s c}(0 ; \eta) \cap \beta_{*}\left|\beta(y)=J^{(e)}(\alpha)(y)+c y^{e},\right| c \mid \leq \rho\right\} .
$$

The total asymptotic Gaussian curvature over $\mathcal{D}^{(e)}\left(\alpha_{*} ; \rho\right)$ is, by definition,

$$
\mathscr{M}_{f}\left(\mathcal{D}^{(e)}\left(\alpha_{*} ; \rho\right)\right):=\lim _{\eta \rightarrow 0}\left\{\lim _{c \rightarrow 0} \int_{\mathcal{S}_{c} \cap H_{o r n}^{(e)}\left(\alpha_{*} ; \rho ; \eta\right)} K d S\right\},
$$

where $\mathcal{S}_{c}$ is the level surface $f=c, S$ the surface area, and $K$ the Gaussian curvature.

The total asymptotic Gaussian curvature over an enriched line is, by definition,

$$
\mathscr{M}_{f}\left(\mathcal{L}^{(e)}\left(\alpha_{*}\right)\right):=\lim _{\rho \rightarrow \infty} \mathscr{M}_{f}\left(\mathcal{D}^{(e)}\left(\alpha_{*} ; \rho\right)\right) .
$$

The above definitions are easily extended to the case $e=\infty$, so that

$$
\mathscr{M}_{f}\left(\mathcal{D}^{(\infty)}\left(\alpha_{*}\right)\right)=\mathscr{M}_{f}\left(\mathcal{L}^{(\infty)}\left(\alpha_{*}\right)\right)=\mathscr{M}_{f}\left(\left\{\alpha_{*}\right\}\right)=0 .
$$

Note. The order of integration in (2.19) is vital; if reversed, it is meaningless.

The "real picture" of a horn domain is shown in Fig. 2, as a model for $\mathcal{D}^{(e)}\left(\alpha_{*} ; \rho\right)$.

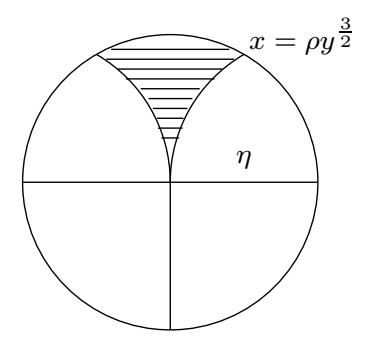

FiguRE 2. Horn Domain $H_{o r n}^{(3 / 2)}\left(0_{*} ; \rho ; \eta\right)$

Attention. In Analytic Geometry, when returning to $(x, y)$ from $(r, \theta)$, there was no problem. Here, however, we have to be more careful when shifting from $\mathbb{C}_{\text {enriched }}$ back to $\mathbb{C}^{2}$. In (2.18), $J^{(e)}(\alpha)$ is a finite series, $\beta_{*}$ is defined globally. If we use infinite series, the germs may not be defined in $D_{i s c}(0 ; \eta)$. Even if we take $J^{(e)}(\alpha)$, and all finite series $\beta$ such that $\beta_{*} \in \mathcal{D}^{(e)}\left(\alpha_{*} ; \rho\right)$, then, since the terms of order $>e$ can have arbitrarily large coefficients, the union of all $\beta_{*}$ would cover the entire disc, except the $z$-axis. The integration (2.19) would be independent of $e$, hence meaningless. The picture in Fig.2 would not be horn-shaped. 
Now, a well-known formula to compute the Milnor number $\mu_{f}$ of $f(z, w)$ is

$$
\mu_{f}=\sum_{j=1}^{m-1}\left[O_{y}\left(f\left(\gamma_{j}(y), y\right)\right)-1\right], \quad \gamma_{j} \text { as in (2.10). }
$$

In particular, $\mu_{f}=\infty$ if 0 is not an isolated singularity.

We define the Milnor number of $f$ on a gradient canyon $\mathcal{G C}\left(\gamma_{*}\right), d_{g r}\left(\gamma_{*}\right)<\infty$, to be

$$
\mu_{f}\left(\mathcal{G C}\left(\gamma_{*}\right)\right):=\sum_{j}\left[O_{y}\left(f\left(\gamma_{j}(y), y\right)\right)-1\right]
$$

the sum being taken over all $j, 1 \leq j \leq m-1$, such that $\gamma_{j *} \in \mathcal{G C}\left(\gamma_{*}\right)$.

The multiplicity of $\mathcal{G C}\left(\gamma_{*}\right)$ is, by definition,

$$
m\left(\mathcal{G C}\left(\gamma_{*}\right)\right):=\sharp\left\{j \mid 1 \leq j \leq m-1, \gamma_{j *} \in \mathcal{G C}\left(\gamma_{*}\right)\right\} .
$$

Of course, combining (2.22) and (2.23), we have

$$
\mu_{f}\left(\mathcal{G C}\left(\gamma_{*}\right)\right)+m\left(\mathcal{G C}\left(\gamma_{*}\right)\right)=\sum_{j} O_{y}\left(f\left(\gamma_{j}(y), y\right)\right),
$$

summing over those $j$ as in (2.22).

Example 2.9. Take $f(z, w)=z^{4}-4 z w^{4}-w^{5}$. The polars are $y^{4 / 3}, \omega y^{4 / 3}$ and $\omega^{2} y^{4 / 3}$, where $\omega^{3}=1$. There is only one gradient canyon, having degree $4 / 3$. We have

$$
\mu_{f}\left(\mathcal{G C}\left(\gamma_{*}\right)\right)=3 \times(5-1)=12, \quad m\left(\mathcal{G C}\left(\gamma_{*}\right)\right)=3 .
$$

Theorem C. (Compare [10], [1.) Let $\gamma_{*}$ be a polar, $1<d_{g r}\left(\gamma_{*}\right) \leq \infty$. Then

$$
\mathscr{M}_{f}\left(\mathcal{G C}\left(\gamma_{*}\right)\right)= \begin{cases}2 \pi\left[\mu_{f}\left(\mathcal{G C}\left(\gamma_{*}\right)\right)+m\left(\mathcal{G C}\left(\gamma_{*}\right)\right],\right. & 1<d_{g r}\left(\gamma_{*}\right)<\infty \\ 0, & d_{g r}\left(\gamma_{*}\right)=\infty\end{cases}
$$

Thus, in Example 2.9, the total asymptotic Gaussian curvature is

$$
\mathscr{M}_{f}\left(\mathcal{G C}\left(\gamma_{*}\right)\right)=2 \pi[12+3]=30 \pi .
$$

Next, for convenience, let us permute the indices, if necessary, so that

$$
\left\{\mathcal{G C}\left(\gamma_{1 *}\right), \ldots, \mathcal{G C}\left(\gamma_{s *}\right)\right\}
$$

is the set of all minimal gradient canyons of degree $>1$. By (2.8), they are mutually disjoint.

Theorem D. (Compare [1.) The "Dirac phenomenon" appears on every infinitesimal line.

That is to say, let $\mathcal{L}:=\mathcal{L}^{(e)}, e>1$, be given, then

$$
\mathscr{M}_{f}(\mathcal{L}) \neq 0 \Longleftrightarrow \mathcal{G C}\left(\gamma_{j *}\right) \subseteq \mathcal{L} \text { for some } j, 1 \leq j \leq s ;
$$

and in this case

$$
\mathscr{M}_{f}(\mathcal{L})=\sum_{j} \mathscr{M}_{f}\left(\mathcal{G C}\left(\gamma_{j *}\right)\right)
$$

summing over all $j$ such that $\mathcal{G C}\left(\gamma_{j *}\right) \subseteq \mathcal{L}, 1 \leq j \leq s$.

For $e=1$, we have

$$
\mathscr{M}_{f}\left(\mathbb{C}_{\text {enriched }}\right)=2 \pi m(r-1)+\sum_{j=1}^{s} \mathscr{M}_{f}\left(\mathcal{G C}\left(\gamma_{j *}\right)\right) \text {, r as in 2.17). }
$$

In particular, if $f(z, w)$ has exactly $r$ distinct roots $\zeta_{i}$ in (2.10), then

$$
\mathscr{M}_{f}\left(\mathbb{C}_{\text {enriched }}\right)=2 \pi m(r-1) \text {. }
$$


Remark 2.10. Using Carathéodory's Extension Theorem, we can extend $\mathscr{M}_{f}$ to a measure on the $\sigma$-algebra generated by the discs (see Remark 6.9). In particular,

$$
\mathscr{M}_{f}\left(\mathcal{D}^{(e)}\left(\alpha_{*} ; 0\right)\right):=\lim _{\rho \rightarrow 0} \mathscr{M}_{f}\left(\mathcal{D}^{(e)}\left(\alpha_{*} ; \rho\right)\right),
$$

which need not be 0 , see (6.22). Then (2.26) asserts that the measure of $\mathcal{L}^{(e)}$ is concentrated in the gradient canyons contained therein, whence referred to as the "Dirac phenomenon".

Corollary 2.11. ([10], [1].) If 0 is an isolated singularity, then

$$
\mathscr{M}_{f}\left(\mathbb{C}_{\text {enriched }}\right)=2 \pi\left[\mu_{f}+O(f)-1\right] .
$$

We now state Theorem $E$, to be proved in $₫ 7$, Let us decompose $f_{z}$ in $\mathbb{C}\{z, w\}$ :

$$
f_{z}(z, w)=\text { unit } \cdot p_{1}(z, w)^{e_{1}} \cdots p_{\tau}(z, w)^{e_{\tau}}, \quad e_{k} \geq 1,
$$

where $p_{k}(z, w) \in \mathbb{C}\{z, w\}$ are the (distinct) irreducible factors, mini-regular in $z$.

For convenience, let us permute the indices of $\left\{\gamma_{j}\right\}$, if necessary, so that

$$
p_{k}\left(\gamma_{k}(w), w\right)=0, \quad 1 \leq k \leq \tau .
$$

That is, $\gamma_{k}$, together with the conjugates, are the Newton-Puiseux roots of $p_{k}(z, w)$.

Take a set of generic numbers $\epsilon_{k, i}$ :

$$
\epsilon:=\left\{\epsilon_{k, i}\left|1 \leq i \leq e_{k}, 1 \leq k \leq \tau,\right| \epsilon_{k, i} \mid \text { sufficiently small }\right\} .
$$

Take a fixed $k$. Let $\hat{\gamma}_{k}(y)$ denote $\gamma_{k}(y)$ with all terms $y^{e}, e>d_{g r}\left(\gamma_{k}\right)$, deleted. We then perturb $\hat{\gamma}_{k}(y)$ to

$$
\gamma_{k, i}^{(\epsilon)}(y):=\hat{\gamma}_{k}(y)+\epsilon_{k, i} y^{d_{k}} \in \mathcal{G}\left(\gamma_{k}\right), \quad d_{k}:=d_{g r}\left(\gamma_{k}\right), \quad 1 \leq i \leq e_{k} .
$$

For each $i, \gamma_{k, i}^{(\epsilon)}$ generates an irreducible function germ

$$
p_{k, i}^{(\epsilon)}(z, w) \in \mathbb{C}\{z, w\}, \quad p_{k, i}^{(\epsilon)}\left(\gamma_{k, i}^{(\epsilon)}(y), y\right)=0 .
$$

We then define

$$
P_{k}^{(\epsilon)}(z, w):=\prod_{i=1}^{e_{k}} p_{k, i}^{(\epsilon)}(z, w), \quad f_{z}^{(\epsilon)}(z, w):=\prod_{k=1}^{\tau} P_{k}^{(\epsilon)}(z, w) .
$$

The Newton-Puiseux roots of $f_{z}^{(\epsilon)}(z, w)$ are mutually distinct.

Now let $S^{3} \subset \mathbb{C}^{2}$ denote a 3 -sphere centered at 0 with sufficiently small radius,

$$
V_{k, i}^{(\epsilon)}:=\left\{(z, w) \mid p_{k, i}^{(\epsilon)}(z, w)=0\right\}, \quad K_{k, i}^{(\epsilon)}:=V_{k, i}^{(\epsilon)} \cap S^{3} ;
$$

each $K_{k, i}^{(\epsilon)}$ is an iterated torus knot ([3], [11], [15]). We call

$$
\mathscr{C}_{k}^{(\epsilon)}:=\left\{K_{k, i}^{(\epsilon)} \mid 1 \leq i \leq e_{k}\right\}, \quad \mathscr{N}^{(\epsilon)}:=\left\{\mathscr{C}_{k}^{(\epsilon)} \mid 1 \leq k \leq \tau\right\},
$$

a cable of knots, and a network of cables, respectively.

Next, take another set of generic numbers $\delta:=\left\{\delta_{k, i}\right\}$. Define $p_{k, i}^{(\delta)}, f_{z}^{(\delta)}, K_{k, i}^{(\delta)}, \mathscr{C}_{k}^{(\delta)}, \mathscr{N}^{(\delta)}$. 
Let $\mathscr{L}\left(K_{k, i}^{(\epsilon)}, K_{l, j}^{(\delta)}\right)$ denote the linking number of two knots in the usual sense. We call

$$
\mathscr{L}\left(\mathscr{C}_{k}^{(\epsilon)}, \mathscr{C}_{l}^{(\delta)}\right):=\sum_{i=1}^{e_{k}} \sum_{j=1}^{e_{l}} \mathscr{L}\left(K_{k, i}^{(\epsilon)}, K_{l, j}^{(\delta)}\right)
$$

the linking number of the cables.

We call $\mathscr{N}^{(\epsilon)}, \mathscr{N}^{(\delta)}$ a twin networks. Their linking number is, by definition,

$$
\mathscr{L}\left(\mathscr{N}^{(\epsilon)}, \mathscr{N}^{(\delta)}\right):=\sum_{k=1}^{\tau} \sum_{l=1}^{\tau} \mathscr{L}\left(\mathscr{C}_{k}^{(\epsilon)}, \mathscr{C}_{l}^{(\delta)}\right) .
$$

Theorem E. Suppose 0 is an isolated singularity of $f(z, w)$. Then

$$
\mu_{f}=\mathscr{L}\left(\mathscr{N}^{(\epsilon)}, \mathscr{N}^{(\delta)}\right) .
$$

This formula provides a geometric interpretation of $\mu_{f}$ using gradient canyons.

\section{Newton Polygons Relative to An Arc}

Two lemmas are presented in this section. The first is used to compute $d_{g r}\left(\gamma_{*}\right)$. The second is pivotal for proving of Lemma 4.1, the latter is pivotal for proving Theorem A.

Let $\gamma$ be a given polar in (2.10),$d_{g r}(\gamma)<\infty$, i.e., $f_{z}(\gamma(y), y) \equiv 0, f(\gamma(y), y) \not \equiv 0$.

We can apply a unitary transformation, if necessary, so that $\gamma \in \mathbb{F}_{1^{+}}, T\left(\gamma_{*}\right)=[0: 1]$.

We then change variables (formally):

$$
Z:=z-\gamma(w), \quad W:=w, \quad F(Z, W):=f(Z+\gamma(W), W) .
$$

Since $\gamma \in \mathbb{F}_{1^{+}}$, i.e., $O(\gamma)>1$, it is easy to see that

$$
\left\|\operatorname{Grad}_{z, w} f\right\| \sim\left\|\operatorname{Grad}_{Z, W} F\right\|, \quad \Delta_{f}(z, w)=\Delta_{F}(Z, W)+\gamma^{\prime \prime}(W) F_{Z}^{3},
$$

and accordingly,

$$
K(z, w) \sim \frac{2\left|\Delta_{F}+\gamma^{\prime \prime} F_{Z}^{3}\right|^{2}}{\left(\left|F_{Z}\right|^{2}+\left|F_{W}\right|^{2}\right)^{3}}, \quad O\left(\gamma^{\prime \prime}\right)>-1 .
$$

(As before, $A \sim B$ means $A / B \rightarrow 1$ as $(z, w) \rightarrow 0$.)

The Newton polygon $\mathcal{N} \mathcal{P}(F)$ is defined in the usual way, as follows. Let us write

$$
F(Z, W)=\sum c_{i q} Z^{i} W^{q}, \quad c_{i q} \neq 0, \quad(i, q) \in \mathbb{Z} \times \mathbb{Q} .
$$

A monomial term with $c_{i q} \neq 0$ is represented by a "Newton dot" at $(i, q)$. We shall simply call it a dot of $F$. The boundary of the convex hull generated by $\{(i+u, q+v) \mid u, v \geq 0\}$, for all dots $(i, q)$, is the Newton Polygon $\mathcal{N} \mathcal{P}(F)$, having edges $E_{i}$ and angles $\theta_{i}$, as shown in Fig.3. In particular, $E_{0}$ is the half-line $[m, \infty)$ on the $Z$-axis. (In [6], 7], this is called the Newton Polygon of $f$ relative to $\gamma$, denoted by $\mathcal{N} \mathcal{P}(f, \gamma)$.)

For a line in $\mathbb{R}^{2}$ joining $(u, 0)$ and $(0, v)$, let us call $v / u$ its co-slope. Thus

$$
\text { co-slope of } E_{s}=\tan \theta_{s} \text {. }
$$

Some elementary, but useful, facts are:

- If $i \geq 1$, then $(i, q)$ is a dot of $F$ if and only if $(i-1, q)$ is one of $F_{Z}$.

- $F_{Z}(0, W) \equiv 0 ; F_{Z}$ has no dot of the form $(0, q) ; F$ has no dot of the form $(1, q)$. 
- Since $f(\gamma(w), w) \not \equiv 0$, we know $F(0, W) \not \equiv 0$. Let us write

$$
F(0, W)=a W^{h}+\cdots, \quad a \neq 0, h:=O_{W}(F(0, W)) \in \mathbb{Q} .
$$

Then $(0, h)$ is a vertex of $\mathcal{N} \mathcal{P}(F),(0, h-1)$ is one of $\mathcal{N} \mathcal{P}\left(F_{W}\right)$. (See Fig. 4 .)

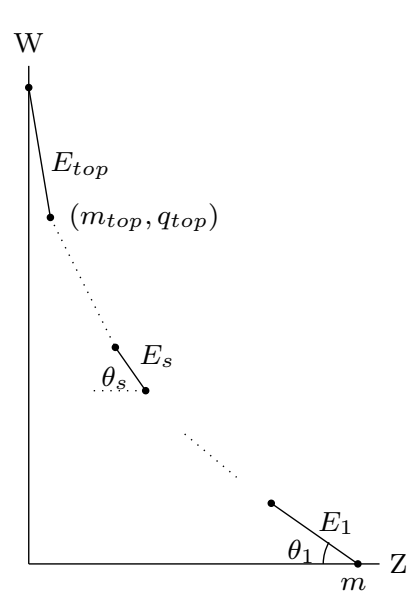

Figure $3 . \mathcal{N} \mathcal{P}(F)$

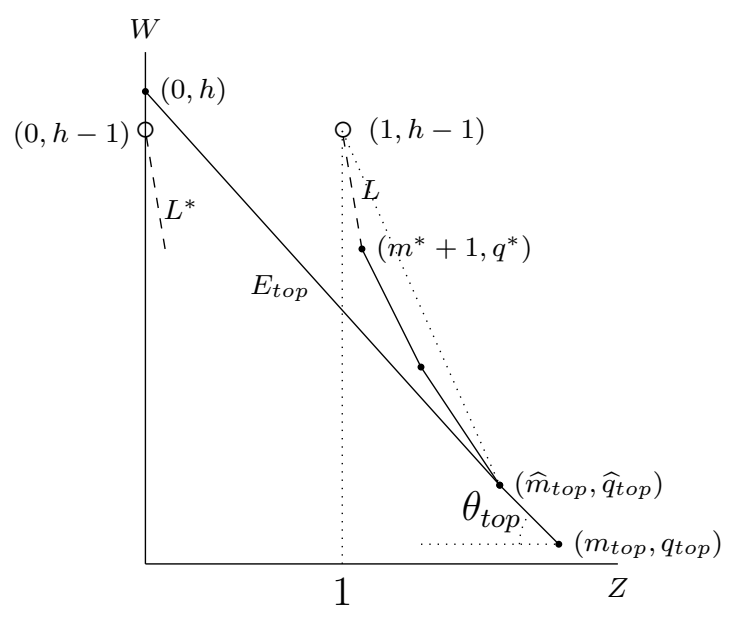

Figure 4. $\mathcal{N} \mathcal{P}(F)$ vs $\mathcal{N} \mathcal{P}\left(F_{Z}\right)$

Notations 3.1. Let $E_{\text {top }}$ denote the edge whose left vertex is $(0, h), h$ as in (3.4), and right vertex is $\left(m_{t o p}, q_{t o p}\right)$, as shown in the figures. We call it the top edge; the angle is $\theta_{\text {top }}$.

Let $\left(\widehat{m}_{t o p}, \widehat{q}_{t o p}\right) \neq(0, h)$ be the dot of $F$ on $E_{\text {top }}$ which is closest to $(0, h)$. (Of course, $\left(\widehat{m}_{t o p}, \widehat{q}_{t o p}\right)$ may coincide with $\left(m_{t o p}, q_{t o p}\right)$.) Then, clearly,

$$
2 \leq \widehat{m}_{t o p} \leq m_{t o p}, \quad \frac{h-\widehat{q}_{t o p}}{\widehat{m}_{t o p}}=\frac{h-q_{t o p}}{m_{t o p}}=\tan \theta_{t o p}
$$

Now we draw a line $L$ through $(1, h-1)$ with the following two properties:

- If $\left(m^{\prime}, q^{\prime}\right)$ is a dot of $F_{Z}$, then $\left(m^{\prime}+1, q^{\prime}\right)$ lies on or above $L$;

- There exists a dot $\left(m^{*}, q^{*}\right)$ of $F_{Z}$ such that $\left(m^{*}+1, q^{*}\right) \in L$. (Of course, $\left(m^{*}+1, q^{*}\right)$ may coincide with $\left(\widehat{m}_{t o p}, \widehat{q}_{t o p}\right)$.)

Lemma 3.2. Let $\sigma^{*}$ denote the co-slope of L. Then

$$
\text { (i) } d_{g r}(\gamma)=\sigma^{*} ;(i i) \sigma^{*} \geq \tan \theta_{\text {top }} ;(\text { iii }) \sigma^{*}=\tan \theta_{\text {top }} \Leftrightarrow \tan \theta_{\text {top }}=1 .
$$

All dots of $F_{W}$ lie on or above $L^{*}, L^{*}$ being the line through $(0, h-1)$ parallel to $L$.

In the case $\tan \theta_{\text {top }}>1,(0, h-1)$ is the only dot of $F_{W}$ on $L^{*}$.

In Example (2.5),$L$ is the line joining $(1, n-1)$ and $(m, 0)$, hence $d=\frac{n-1}{m-1}$.

Example. For $F(Z, W)=Z^{4}+Z^{3} W^{27}+Z^{2} W^{63}-W^{100}$ and $\gamma=0, \mathcal{N} \mathcal{P}(F)$ has only two vertices $(4,0),(0,100)$, while $\mathcal{N} \mathcal{P}\left(F_{Z}\right)$ has three: $(3,0),(2,27),(1,63)$. The latter two and $(0,99)$ are collinear, spanning $L^{*} ; h=100, \sigma^{*}=(99-27) / 2=36$. 
Notations 3.3. Take $e \geq 1$. Let $\omega(e)$ denote the weight system: $\omega(Z)=e, \omega(W)=1$.

Let $G\left(Z, W^{1 / N}\right) \in \mathbb{C}\left\{Z, W^{1 / N}\right\}$ be given. Consider its weighted Taylor expansion relative to this weight. We shall denote the weighted initial form by $\mathcal{I}_{\omega(e)}(G)(Z, W)$, or simply $\mathcal{I}_{\omega}(G)$ when there is no confusion.

If $\mathcal{I}_{\omega}(G)=\sum a_{i j} Z^{i} W^{j / N}$, the weighted order of $G$ is $O_{\omega}(G):=i e+\frac{j}{N}$.

Proof. Note that $(i i)$ and $(i i i)$ are clearly true, since $(1, h-1)$ lies on or above $E_{\text {top }}$.

Next, if $(i, q)$ is a dot of $F_{W}$, then $(i, q+1)$ is one of $F$, lying on or above $E_{\text {top }}$. Hence, by (ii), all dots of $F_{W}$ lie on or above $L^{*}$.

It also follows that if $\tan \theta_{t o p}>1$, then $(0, h-1)$ is the only $\operatorname{dot}$ of $F_{W}$ on $L^{*}$.

Now we show $(i)$.

It is easy to see that if $\tan \theta_{\text {top }}=1$, then $d_{g r}(\gamma)=1$.

It remains to consider the case $\sigma^{*}>1$. Since $\gamma$ is a polar,

$$
O_{y}(\|\operatorname{Grad} f(\gamma(y), y)\|)=O_{W}\left(F_{W}(0, W)\right)=h-1 .
$$

Let us first take weight $\omega:=\omega(e)$ where $e \geq \sigma^{*}$. In this case, since $\sigma^{*}>1$,

$$
\mathcal{I}_{\omega}\left(F_{W}\right)(Z, W)=a h W^{h-1}, \quad O_{\omega}\left(F_{Z}\right) \geq h-1 ;
$$

where $a, h$ are as in (3.4), $a h \neq 0$. Hence for all $u \in \mathbb{C}$,

$$
O_{W}\left(F_{W}\left(u W^{e}, W\right)\right)=h-1, \quad O_{W}\left(F_{Z}\left(u W^{e}, W\right)\right) \geq h-1 .
$$

It follows that $d_{g r}(\gamma) \leq \sigma^{*}$. It remains to show that $\sigma^{*}>d_{g r}(\gamma)$ is impossible.

Let us take $\omega(e)$ with $e<\sigma^{*}$. Note that $\left(m^{*}, q^{*}\right)$ is a dot of $F_{Z}$ on $L^{*}$, where $\left(m^{*}+1, q^{*}\right)$ is shown in Fig 4. Hence, for generic $u$,

$$
O_{W}\left(F_{Z}\left(u W^{e}, W\right)\right)<h-1, \quad O_{W}\left(\left\|\operatorname{Grad} F\left(u W^{e}, W\right)\right\|\right)<h-1 .
$$

Thus, by (3.6), we must have $d_{g r}(\gamma)>e$. This completes the proof.

For the next lemma, let us expand $\Delta_{F}$ in (3.2):

$$
\Delta_{F}=-F_{Z Z} F_{W}^{2}-F_{Z}^{2} F_{W W}+2 F_{Z} F_{W} F_{Z W} .
$$

Lemma 3.4. Let $\gamma$ be a given polar, $1<d:=d_{g r}(\gamma)<\infty, \omega:=\omega(e)$ a weight system. If $e \geq d$, then the following is true:

$$
O_{\omega}\left(F_{Z Z} F_{W}^{2}\right)<\min \left\{O_{\omega}\left(F_{Z}^{2} F_{W W}\right), O_{\omega}\left(F_{Z} F_{W} F_{Z W}\right), O_{\omega}\left(\gamma^{\prime \prime} F_{Z}^{3}\right)\right\} .
$$

It follows that

$$
\mathcal{I}_{\omega}\left(F_{Z Z} F_{W}^{2}\right)=\mathcal{I}_{\omega}\left(\Delta_{F}\right)=\mathcal{I}_{\omega}\left(\Delta_{F}+\gamma^{\prime \prime} F_{Z}^{3}\right) .
$$

If we merely assume $e \geq \tan \theta_{1}$, then a weaker statement is true:

$$
O_{\omega}\left(F_{Z Z} F_{W}^{2}\right)=O_{\omega}\left(\Delta_{F}\right), O_{\omega}\left(\Delta_{F}+\gamma^{\prime \prime} F_{Z}^{3}\right)=\min \left\{O_{\omega}\left(\Delta_{F}\right), O_{\omega}\left(\gamma^{\prime \prime} F_{Z}^{3}\right)\right\} .
$$


Proof. Since $F_{Z}$ has no dot on the $W$-axis, we can write

$$
\mathcal{I}_{\omega}\left(F_{Z}\right)(Z, W):=a_{k} Z^{k} W^{q}+\cdots+a_{M} Z^{M} W^{q-(M-k) e}, \quad a_{k} \neq 0 \neq a_{M},
$$

where $1 \leq k \leq M, q \in \mathbb{Q}$. Then, clearly,

$$
O_{\omega}\left(F_{Z}\right)=q+k e, \quad O_{\omega}\left(F_{Z Z}\right)=q+(k-1) e .
$$

Now suppose $e \geq \tan \theta_{\text {top }}$. Let $h, a$ be as in (3.4). In this case,

$$
O_{\omega}(F)=h, \quad O_{\omega}\left(F_{W}\right)=h-1, \quad q+(k+1) e \geq h .
$$

The last inequality holds since $(k+1, q)$ lies on or above $E_{\text {top }}$. Thus, by (3.12), (3.13),

$$
O_{\omega}\left(F_{Z Z} F_{W}^{2}\right)=q+(k-1) e+2(h-1), O_{\omega}\left(\gamma^{\prime \prime} F_{Z}^{3}\right)=[O(\gamma)-2]+3(q+k e) .
$$

We obtain the following:

$$
O_{\omega}\left(F_{Z Z} F_{W}^{2}\right) \leq O_{\omega}\left(F_{Z}^{2} F_{W W}\right)=O_{\omega}\left(F_{Z} F_{W} F_{Z W}\right)=2(q+k e)+(h-2),
$$

where " $\leq$ " can be replaced by " $<$ " if $e>\tan \theta_{\text {top }}$.

In the case $e \geq d$, by Lemma 3.2 , we have

$$
e>\tan \theta_{\text {top }}, \quad q+k e \geq h-1 .
$$

Note that $O\left(\gamma^{\prime \prime}\right)>-1$. Hence, as an immediate consequence of (3.14) and (3.16),

$$
O_{\omega}\left(F_{Z Z} F_{W}^{2}\right)<O_{\omega}\left(\gamma^{\prime \prime} F_{Z}^{3}\right) .
$$

This completes the proof of (3.8), and hence also that of (3.9).

We now prove (3.10). First suppose $e \geq \tan \theta_{t o p}$.

In this case we do not necessarily have the second inequality in (3.16). However, the leading term of

$$
\mathcal{I}_{\omega}\left(F_{Z Z} F_{W}^{2}\right)(Z, W)=k a_{k} a^{2} h^{2} Z^{k-1} W^{q+2(h-1)}+\cdots,
$$

has $Z$-order $k-1$. All other terms in $\mathcal{I}_{\omega}\left(\Delta_{F}\right)$ have $Z$-order $>k-1$.

Hence no cancellation with the leading term of (3.17) can happen in $\Delta_{F}$, the first equality in (3.10) follows from (3.15).

The second equality is also clear. The $Z$-order of $\mathcal{I}_{\omega}\left(\gamma^{\prime \prime} F_{Z}^{3}\right)$ is $3 k$, which is larger than that of $F_{Z Z} F_{W}^{2}$. Hence there is no cancellation,

$$
O_{\omega}\left(\Delta_{F}\right)=O_{\omega}\left(\gamma^{\prime \prime} F_{Z}^{3}\right) \Longrightarrow O_{\omega}\left(\Delta_{F}+\gamma^{\prime \prime} F_{Z}^{3}\right)=O_{\omega}\left(\Delta_{F}\right) .
$$

On the other hand, the following is obviously true:

$$
O_{\omega}\left(\Delta_{F}\right) \neq O_{\omega}\left(\gamma^{\prime \prime} F_{Z}^{3}\right) \Longrightarrow O_{\omega}\left(\Delta_{F}+\gamma^{\prime \prime} F_{Z}^{3}\right)=\min \left\{O_{\omega}\left(\Delta_{F}\right), O_{\omega}\left(\gamma^{\prime \prime} F_{Z}^{3}\right)\right\} .
$$

We have proved the second equality in (3.10) in the case $e \geq \tan \theta_{\text {top }}$.

Now suppose $\tan \theta_{1} \leq e<\tan \theta_{\text {top. }}$. In this case

$$
O_{\omega}\left(F_{W}\right)=q+(k+1) e-1,
$$

where $q \geq 1$ since $(k+1, q)$ can be at worst the left vertex of $E_{1}$. Hence

$$
O_{\omega}\left(F_{Z Z} F_{W}^{2}\right)=O_{\omega}\left(F_{Z} F_{W} F_{Z W}\right)=3(q+k e)+e-2 \leq O_{\omega}\left(F_{Z}^{2} F_{W W}\right) .
$$

(The last inequality is an equality if $q>1$.) The same $Z$-order argument proves (3.10). 
Example. It can happen that $\gamma^{\prime \prime} F_{Z}^{3}$ dominates $\Delta_{F}$. Take

$$
f(z, w):=\left(z-w^{2}\right)^{m}-w^{n}, \quad \gamma=w^{2}, \quad e=n / m,
$$

where $n>2 m$. We then have

$$
O_{\omega}\left(F_{Z}\right)=\frac{n}{m} \cdot(m-1), O_{\omega}\left(F_{W}\right)=n-1, O_{\omega}\left(\gamma^{\prime \prime} F_{Z}^{3}\right)<O_{\omega}\left(F_{Z Z} F_{W}^{2}\right) .
$$

\section{The Lojasiewicz Exponent Function}

Let $\alpha \in \mathbb{F}$ be given. Take $e \geq 1$. Take a generic $u \in \mathbb{C}$, or an indeterminate. Write

$$
\begin{aligned}
& \left|\Delta_{f}\left(\alpha(y)+u y^{e}, y\right)\right|^{2}:=N_{(\alpha, e)}(u) y^{2 L_{\Delta}(\alpha, e)}+\cdots, N_{(\alpha, e)}(u) \not \equiv 0, \\
& \left\|\operatorname{Grad} f\left(\alpha(y)+u y^{e}, y\right)\right\|^{2}:=D_{(\alpha, e)}(u) y^{2 L_{g r}(\alpha, e)}+\cdots, D_{(\alpha, e)}(u) \not \equiv 0 \text {, }
\end{aligned}
$$

where $N_{(\alpha, e)}(u), D_{(\alpha, e)}(u)$ are real-valued, non-negative, polynomials. We also write

$$
L_{\alpha}(e):=L_{\Delta}(\alpha, e)-3 L_{g r}(\alpha, e), \quad R_{(\alpha, e)}(u):=N_{(\alpha, e)}(u) D_{(\alpha, e)}(u)^{-3} .
$$

Observe that $L_{\Delta}(\alpha, e), L_{g r}(\alpha, e)$ are defined even if $e$ is irrational. Hence they are piecewise linear, continuous, increasing functions of $e$, defined for all $e \in[1, \infty)$.

As in Calculus, we say $\phi(x)$ is increasing (resp. decreasing, resp. strictly decreasing) if

$$
x_{1}<x_{2} \Longrightarrow \phi\left(x_{1}\right) \leq \phi\left(x_{2}\right)\left(\text { resp. } \phi\left(x_{1}\right) \geq \phi\left(x_{2}\right) \text {, resp. } \phi\left(x_{1}\right)>\phi\left(x_{2}\right)\right) \text {. }
$$

We call $L_{\alpha}(e)$ the Lojasiewicz exponent function along $\alpha$. It is piecewise linear, continuous (but not necessarily increasing). Note that $K_{*}$, defined in (1.5), can be written as

$$
K_{*}\left(\eta_{*}\right)=2 R_{(\alpha, e)}(u) \delta^{2 L_{\alpha}(e)}, \quad \eta(y):=\alpha(y)+u y^{e}, \text { u generic. }
$$

Note also that $R_{\gamma}(u)$ and $L_{\gamma}$ defined in (2.13) are special cases:

$$
R_{\gamma}(u)=R_{(\gamma, d)}(u), \quad L_{\gamma}=L_{\gamma}(d), \quad d:=d_{g r}(\gamma) .
$$

Lemma 4.1. Let $\gamma$ be a given polar, $1<d:=d_{g r}(\gamma)<\infty$. Then

(1) $L_{\gamma}(e)>-1$ for $1<e<\tan \theta_{1}, \theta_{1}$ being the first angle of $\mathcal{N} \mathcal{P}(F)$ (Fig.

(2) $L_{\gamma}(e)$ is increasing for $e \in\left(\tan \theta_{1}, \tan \theta_{\text {top }}\right)$;

(3) $L_{\gamma}(e)$ is decreasing for $e \in\left(\tan \theta_{\text {top }}, d\right)$;

(4) $L_{\gamma}(e)$ is strictly decreasing for $e \in(d-\epsilon, d), \epsilon>0$ sufficiently small;

(5) $L_{\gamma}(d)=-d=L_{\gamma}$;

(6) If $O(\alpha-\gamma) \geq d$, then $L_{g r}(\alpha, e)=L_{g r}(\gamma, d)$ and $L_{\alpha}(e)$ is increasing for $e \geq d$.

The above open intervals can be replaced by closed intervals; $L_{\gamma}(q)=-d$ is the absolute minimum of $L_{\gamma}(e), e \in[1, d]$; moreover, $L_{\gamma}(q) \geq-d$ for $q>d$.

Proof. We first prove (6). Let $a, h$ be as in (3.4).

If $O(\alpha-\gamma) \geq d$, then for all $e \geq d$,

$$
D_{(\alpha, e)}(u) \geq|a h|^{2}>0 \forall u, L_{g r}(\alpha, e)=L_{g r}(\gamma, d)=h-1 .
$$

But $L_{\Delta}(\alpha, e)$ is increasing, hence so is $L_{\alpha}(e), e \geq d$.

Let us apply (3.9) with $e=d$. Then (5) follows from:

$$
O_{\omega(d)}\left(F_{W}\right)=h-1, \quad L_{\Delta}(\gamma, d)=3(h-1)-d, \quad L_{g r}(\gamma, d)=h-1 .
$$


To prove (4), take weight $\omega(d)$, and write expression (3.11). We then consider $\omega(d-\epsilon)$, where $\epsilon>0$ is sufficiently small. We obviously have

$$
\mathcal{I}_{\omega(d-\epsilon)}\left(F_{Z}\right)=a_{M} Z^{M} W^{q-(M-k) d}, \quad \mathcal{I}_{\omega(d-\epsilon)}\left(F_{Z Z}\right)=a_{M} M Z^{M-1} W^{q-(M-k) d} .
$$

Moreover, (3.9) remains true when $\omega(d)$ is replaced by $\omega(d-\epsilon)$ :

$$
\mathcal{I}_{\omega(d-\epsilon)}\left(F_{Z Z} F_{W}^{2}\right)=\mathcal{I}_{\omega(d-\epsilon)}\left(\Delta_{F}\right)=\mathcal{I}_{\omega(d-\epsilon)}\left(\Delta_{F}+\gamma^{\prime \prime} F_{Z}^{3}\right) .
$$

It follows that

$$
L_{\Delta}(\gamma, d-\epsilon)=L_{\Delta}(\gamma, d)-(M-1) \epsilon, \quad L_{g r}(\gamma, d-\epsilon)=L_{g r}(\gamma, d)-M \epsilon,
$$

and then

$$
L_{\gamma}(d-\epsilon)-L_{\gamma}(d)=(2 M+1) \epsilon>0, \quad L_{\gamma}(d-\epsilon)>L_{\gamma}(d) .
$$

To prove (3), take any $e, \tan \theta_{\text {top }}<e<d, \omega:=\omega(e)$. Let us write

$$
N:=\operatorname{deg} \mathcal{I}_{\omega}\left(\Delta_{F}+\gamma^{\prime \prime} F_{Z}^{3}\right)(Z, 1) \quad(\text { degree of a polynomial in } Z \text { ). }
$$

Let $\epsilon>0$ be sufficiently small, then, like (4.4), we have

$$
L_{\Delta}(\gamma, e-\epsilon)=L_{\Delta}(\gamma, e)-N \epsilon, \quad L_{g r}(\gamma, e-\epsilon)=L_{g r}(\gamma, e)-M \epsilon .
$$

Hence it suffices to show that $N \leq 3 M$. Let us write $p(Z), p_{1}(Z), p_{2}(Z)$ for

$$
\mathcal{I}_{\omega}\left(F_{Z Z} F_{W}^{2}\right)(Z, 1), \quad \mathcal{I}_{\omega}\left(F_{Z}^{2} F_{W W}\right)(Z, 1), \quad \mathcal{I}_{\omega}\left(F_{Z} F_{W} F_{Z W}\right)(Z, 1)
$$

respectively. The first equality in (3.10) implies that

$$
\mathcal{I}_{\omega}\left(\Delta_{F}\right)(Z, 1)=p(Z)+c_{1} p_{1}(Z)+c_{2} p_{2}(Z), \quad c_{1}, c_{2} \in \mathbb{C} \text { (possibly zero). }
$$

The second equality in (3.10) implies that

$$
\mathcal{I}_{\omega}\left(\Delta_{F}+\gamma^{\prime \prime} F_{Z}^{3}\right)(Z, 1)=c_{3} \mathcal{I}_{\omega}\left(\Delta_{F}\right)(Z, 1)+c_{4} \mathcal{I}_{\omega}\left(F_{Z}^{3}\right)(Z, 1), \quad\left(c_{3}, c_{4}\right) \neq(0,0) .
$$

The right-hand-side has degree $\leq 3 M$ since $\mathcal{I}_{\omega}\left(F_{W}\right)=a h W^{h-1}$. Hence $N \leq 3 M$.

To prove (2), let us take $e, \tan \theta_{1}<e<\tan \theta_{\text {top }}$, and write (compare (3.11))

$$
\mathcal{I}_{\omega}(F)(Z, W)=a Z^{M+1} W^{l}+a^{\prime} Z^{M} W^{l+e}+\cdots, \quad a \neq 0, \quad \omega=\omega(e) .
$$

Since $e>\tan \theta_{1},(M+1, l)$ cannot be the vertex $(m, 0)$. Hence $l>0$, and

$$
\mathcal{I}_{\omega}\left(F_{W}\right)(Z, W)=l a Z^{M+1} W^{l-1}+\cdots,
$$

where, since $e<\tan \theta_{\text {top }}$, we must have $M+1>0$, and then

$$
\mathcal{I}_{\omega}\left(F_{Z}\right)(Z, W)=a(M+1) Z^{M} W^{l}+\cdots \text {. }
$$

Since $e>1$, an immediate consequence of (4.6) and (4.7) is

$$
O_{W}\left(F_{Z}\left(u W^{e}, W\right)\right)<O_{W}\left(F_{W}\left(u W^{e}, W\right)\right),
$$

and hence

$$
O_{W}\left(\frac{\left|\gamma^{\prime \prime} F_{Z}^{3}\right|^{2}}{\left(\left|F_{Z}\right|^{2}+\left|F_{W}\right|^{2}\right)^{3}}\right)=2 O_{W}\left(\gamma^{\prime \prime}\right) \text {, a constant. }
$$

Now, since $\gamma$ is a polar, we actually have $M+1 \geq 2$. Hence

$$
\mathcal{I}_{\omega}\left(F_{Z Z}\right)(Z, W)=a M(M+1) Z^{M-1} W^{l}+\cdots .
$$


Similarly, we can write down the formulas for $\mathcal{I}_{\omega}\left(F_{Z W}\right)$ and $\mathcal{I}_{\omega}\left(F_{W W}\right)$.

An easy calculation of the determinant $\Delta_{F}$ gives

$$
\mathcal{I}_{\omega}\left(\Delta_{F}\right)(Z, W)=l a^{3}(M+1)(M+l+1) Z^{3 M+1} W^{3 l-2}+\cdots,
$$

and then we have

$$
\operatorname{deg} \mathcal{I}_{\omega}\left(\Delta_{F}\right)(Z, 1)=3 M+1>3 M=\operatorname{deg} \mathcal{I}_{\omega}\left(\gamma^{\prime \prime} F_{Z}^{3}\right)(Z, 1) .
$$

It follows from this inequality that

$$
\mathcal{I}_{\omega}\left(\Delta_{F}+\gamma^{\prime \prime} F_{Z}^{3}\right)= \begin{cases}\mathcal{I}_{\omega}\left(\Delta_{F}\right) & \text { if } O_{\omega}\left(\Delta_{F}\right)<O_{\omega}\left(\gamma^{\prime \prime} F_{Z}^{3}\right) \\ \mathcal{I}_{\omega}\left(\gamma^{\prime \prime} F_{Z}^{3}\right) & \text { if } O_{\omega}\left(\Delta_{F}\right)>O_{\omega}\left(\gamma^{\prime \prime} F_{Z}^{3}\right) \\ \mathcal{I}_{\omega}\left(\Delta_{F}\right)+\mathcal{I}_{\omega}\left(\gamma^{\prime \prime} F_{Z}^{3}\right) & \text { if } O_{\omega}\left(\Delta_{F}\right)=O_{\omega}\left(\gamma^{\prime \prime} F_{Z}^{3}\right)\end{cases}
$$

and hence in every case we have

$$
\operatorname{deg} \mathcal{I}_{\omega}\left(\Delta_{F}+\gamma^{\prime \prime} F_{Z}^{3}\right)(Z, 1) \geq \operatorname{deg} \mathcal{I}_{\omega}\left(\gamma^{\prime \prime} F_{Z}^{3}\right)(Z, 1) .
$$

Now, using (4.8) and the same argument as in the proof of (4), we find

$$
L_{\gamma}(e) \geq L_{\gamma}(e-\epsilon), \quad \epsilon>0 \text { sufficiently small. }
$$

It remains to prove (1). This case involves only the vertex $(m, 0)$ :

$$
O_{\omega}(F)=m e, \quad O_{\omega}\left(F_{Z}\right)=(m-1) e, \quad O_{\omega}\left(F_{W}\right)>m e-1 .
$$

It follows that

$$
O_{\omega}\left(\Delta_{F}\right)-3 O_{\omega}\left(F_{Z}\right)>e-2>-1, \quad O_{\omega}\left(\gamma^{\prime \prime} F_{Z}^{3}\right)-3 O_{\omega}\left(F_{Z}\right)=O\left(\gamma^{\prime \prime}\right)>-1 .
$$

This completes the proof of Lemma 4.1.

\section{Proofs of Theorems A And B}

Let us prove Lemma 2.7. First, consider the case $d:=d_{g r}(\gamma)>1$. We already know $L_{\gamma}=-d$ in Lemma 4.1. Moreover, $R_{\gamma}(u)$ is clearly defined for all $u$ since

$$
D_{(\gamma, d)}(u) \geq|a h|^{2}>0, \quad a, h \text { as in (3.4). }
$$

Let $N, M$ be as in (3.11) and (4.5), where $e=d$. Then, by (3.8),

$$
N=\operatorname{deg} \mathcal{I}_{\omega(d)}\left(F_{Z Z} F_{W}^{2}\right)(u, 1)=M-1<3 M=3 \operatorname{deg} \mathcal{I}_{\omega(d)}\left(F_{Z}\right)(u, 1) .
$$

Hence $\lim _{u \rightarrow \infty} R_{\gamma}(u)=0$.

Now suppose $d=1$. For simplicity, we write $H:=H_{m}(z, w)$. By Euler's Theorem,

$$
\Delta_{H}(z, w)=-\frac{m}{m-1} \cdot H \cdot \operatorname{Hess}(H), \quad \operatorname{Hess}(H):=\left|\begin{array}{ll}
H_{z z} & H_{z w} \\
H_{w z} & H_{w w}
\end{array}\right| .
$$

Note that $H_{m}(z, w)$ has at least two different factors, for otherwise we would have

$$
H_{m}(z, w)=c\left(z-u_{0} w\right)^{m}, \quad d_{g r}(\gamma)>1,
$$

a contradiction. It is easy to see that, as a consequence,

$$
\operatorname{Hess}(H) \not \equiv 0, \quad \Delta_{H} \not \equiv 0 .
$$


Take any $u_{0}$. If $H\left(u_{0}, 1\right) \neq 0$, then $\operatorname{Grad} H\left(u_{0}, 1\right) \neq 0, R_{\gamma}\left(u_{0}\right)$ is clearly defined.

If $z-u_{0} w$ is a factor of $H$ of order $k, k \geq 1$, then it is one of $\Delta_{H}$ of order $3 k-2$,

$$
\Delta_{H}(u, 1)=\left(u-u_{0}\right)^{3 k-2} Q_{1}(u), \quad|\operatorname{Grad} H(u, 1)|=\left|u-u_{0}\right|^{k-1} Q_{2}(u),
$$

where $Q_{1}\left(u_{0}\right) Q_{2}\left(u_{0}\right) \neq 0, u$ near $u_{0}$. Hence $\left|u-u_{0}\right|^{2}$ divides $R_{\gamma}(u), R_{\gamma}\left(u_{0}\right)=0$.

Thus $R_{\gamma}(u)$ is defined for all $u$. It remains to show $L_{\gamma}=-1$ and $\lim _{u \rightarrow \infty} R_{\gamma}(u)=0$.

We can assume $\gamma \in \mathbb{F}_{1^{+}}$. In terms of $(Z, W)$ we have

$$
O_{\omega}\left(\Delta_{H}\right)=3 m-4<O_{\omega}\left(\gamma^{\prime \prime} F_{Z}^{3}\right)=3(m-1)+O(\gamma)-2 .
$$

Hence

$$
O_{\omega}\left(\Delta_{H}+\gamma^{\prime \prime} F_{Z}^{3}\right)=3 m-4, \quad L_{\gamma}=(3 m-4)-3(m-1)=-1 .
$$

We also have $\lim _{u \rightarrow \infty} R_{\gamma}(u)=0$, since

$$
\operatorname{deg} \Delta_{H}(u, 1) \leq 3 m-4<3(m-1)=3 \operatorname{deg} H_{Z}(u, 1) .
$$

Now we first prove Theorem B, after which we prove Theorem $A$.

Let $\mathcal{G C}\left(\gamma_{*}\right)$ be given, $1<d_{g r}\left(\gamma_{*}\right)<\infty$. Take $\gamma_{j}$ in (2.10), and suppose

$$
\mathcal{G C}\left(\gamma_{j *}\right) \subseteq \mathcal{G C}\left(\gamma_{*}\right), \quad 1<d_{g r}\left(\gamma_{j *}\right) \leq \infty .
$$

Amongst the conjugates of $\gamma_{j}$, there is one, say $\gamma_{k}, \gamma_{j *}=\gamma_{k *}$, such that

$$
O_{y}\left(\gamma-\gamma_{k}\right) \geq d_{g r}\left(\gamma_{*}\right) .
$$

It follows from Lemma 3.2 (i) that

$$
O_{y}\left(f\left(\gamma_{k}(y), y\right)\right)=O_{y}(f(\gamma(y), y)), \quad d_{g r}\left(\gamma_{*}\right)=d_{g r}\left(\gamma_{j *}\right)=d_{g r}\left(\gamma_{k *}\right) .
$$

The inclusion in (5.2) is an equality, $d_{g r}\left(\gamma_{j^{*}}\right)=\infty$ cannot happen, $\mathcal{G C}\left(\gamma_{*}\right)$ is minimal.

Recall that two infinitesimal lines are either disjoint, or one contains the other (see (2.8)). The first statement of Theorem B is proved.

For the remaining statements, let $r$ be as in (2.17). By Theorem 2.1 in [7] (Lemma 3.3 in [6]), there are exactly $r-1$ polars $\gamma_{j}$ (counting multiplicities), for each of which

$$
O\left(\gamma_{j}-\zeta_{i}\right)=1, \quad 1 \leq i \leq m, \zeta_{i} \text { as in (2.10). }
$$

Thus, if $r>1$, then, by (3.5) $), d_{g r}\left(\gamma_{j}\right)=1, \mathcal{G C}\left(\gamma_{j *}\right)=\mathbb{C}_{\text {enriched }}$ is a gradient canyon.

If $r=1$, then, by (3.5), $d_{g r}(\gamma)>1$ for all polar $\gamma, \mathbb{C}_{\text {enriched }}$ is not a gradient canyon.

Finally, suppose $1<r \leq m$.

For the roots $\zeta_{i}$ of $f, J^{(1)}\left(\zeta_{i}\right), 1 \leq i \leq m$, are the roots of $H_{m}(z, w)$. Hence if $f$ has more than $r$ distinct roots, then there exist two roots $\zeta_{i}, \zeta_{k}$ such that

$$
1<O\left(\zeta_{i}(y)-\zeta_{k}(y)\right)<\infty
$$

Then, by the same theorem as above, there is a polar $\gamma_{j}$ such that

$$
1<O\left(\zeta_{i}-\zeta_{k}\right)=O\left(\zeta_{i}-\gamma_{j}\right)=O\left(\zeta_{k}-\gamma_{j}\right) \geq O\left(\gamma_{j}-\zeta_{l}\right), \quad 1 \leq l \leq m .
$$

It follows that $1<d_{g r}\left(\gamma_{j *}\right)<\infty, \mathcal{G C}\left(\gamma_{j *}\right)$ is minimal, $\mathbb{C}_{\text {enriched }}$ is not. 
On the other hand, if $f$ has exactly $r$ roots, every polar has $d_{g r}=1$ or $\infty$.

Now we prove Theorem A. We use Lemma 4.1 and the following lemma.

Lemma 5.1. Consider $R_{(\alpha, e)}(u)$ in (4.2). Take $c \in \mathbb{C}$ such that $R_{(\alpha, e)}(c) \neq 0$. Then

$$
\beta(y)=\left[J^{(e)}(\alpha)(y)+c y^{e}\right]+\cdots \Longrightarrow K_{*}\left(\beta_{*}\right)=K_{*}\left(\left(\alpha+c y^{e}\right)_{*}\right) .
$$

Proof. According to (4.2), we can write

$$
R_{(\alpha, e)}(u)=\left|\frac{P(u)}{Q(u)}\right|, \quad P(u), Q(u) \in \mathbb{C}[u],
$$

where $P(c) \neq 0, Q(c) \neq 0$. We then consider the Taylor expansion at $c$,

$$
\frac{P(u)}{Q(u)}=a_{0}+a_{1}(u-c)+\cdots, \quad\left|a_{0}\right|=R_{(\alpha, e)}(c) .
$$

We can write $\beta(y)$ in the form

$$
\beta(y)=\alpha(y)+[c+\nu(y)] y^{e}, \quad \nu(y) \in \mathbb{F}_{0}, \nu(0)=0,
$$

Let us replace $u$ by $\nu(y)+c$ in (5.3), then

$$
R_{(\alpha, e)}(\nu(y)+c)=R_{(\alpha, e)}(c)+\cdots .
$$

It follows that

$$
K(\beta(y), y)=2 R_{(\alpha, e)}(c) y^{L_{\alpha}(e)}+\cdots, \quad K_{*}\left(\beta_{*}\right)=\left(2 R_{(\alpha, e)}(c), L_{\alpha}(e)\right) .
$$

This completes the proof of the Lemma.

Now consider Definition 2.3. Condition (1) follows from the above. The function $R_{\gamma}(u)$, which is not constant, attains a local maximum at $c$, hence (2) holds. Condition (3) follows from Lemma 4.1(4). Hence $\mathcal{D}^{(d)}\left(\beta_{*} ; 0\right)$ is an $\mathrm{A}^{\prime}$ Campo bump.

To show all A'Campo bumps can be obtained in this way, let an A'Campo bump $\mathcal{D}^{(e)}\left(\beta_{*} ; 0\right)$ be given. Take a polar, $\gamma$, such that $O_{y}(\beta-\gamma)$ is maximum. Using Lemma4.1, we see that unless $\beta_{*} \in \mathcal{G C}\left(\gamma_{*}\right)$, there would be a contradiction. The proof of Theorem A is complete.

\section{Proof of Theorems C and D}

We prove Theorem $C$. The case $d_{g r}\left(\gamma_{*}\right)=\infty$ is trivial. We assume $1<d_{g r}\left(\gamma_{*}\right)<\infty$.

Consider $\mathcal{N} \mathcal{P}(F), F(Z, W)$ as in (3.1). In the first place, along $F(Z, W)=c$,

$$
F_{Z} d Z+F_{W} d W=0, \quad d S=\frac{\left|F_{Z}\right|^{2}+\left|F_{W}\right|^{2}}{\left|F_{W}\right|^{2}} \frac{d Z \wedge d \bar{Z}}{-2 \sqrt{-1}} .
$$

Let us set $Z=u W^{d}, d:=d_{g r}(\gamma)>1$, then

$$
d Z=W^{d} d u+(u d) W^{d-1} d W .
$$

Eliminating $d W$,

$$
d Z=\frac{\left(W F_{W}\right)}{W F_{W}+(u d) W^{d} F_{Z}} \cdot W^{d} \cdot d u
$$


Let $h, a$ be as in (3.4). See Fig.4, Then, for generic $u$,

$$
O_{W}\left(F_{Z}\left(u W^{d}, W\right)\right)=h-1,
$$

and for all $u$,

$$
\left.W F_{W}\left(u W^{d}, W\right)=h a W^{h}[1+o(W))\right] .
$$

Convention. Here, as in Calculus, $o(W)$ represents a function $\varphi(W), \varphi(0)=0$.

It follows from the above that for all $u$,

$$
O_{W}\left(W F_{W}\right)=h<h-1+d \leq O_{W}\left(W^{d} F_{Z}\right) .
$$

Hence (6.2) can be rewritten as

$$
d Z=W^{d}[1+o(W)] d u
$$

Now take the weight system $\omega(d): \omega(Z)=d, \omega(W)=1$. Consider the polynomial

$$
p(u):=\mathcal{I}_{\omega(d)}\left(F_{Z}\right)(u, 1) . \quad \text { (As defined in Notation 3.3.) }
$$

Note that, in fact

$$
p(0)=0, \quad \operatorname{deg} p(u)=m\left(\mathcal{G C}\left(\gamma_{*}\right)\right),
$$

the latter was defined in (2.23). By (4.1), (6.3), (3.9),

$$
D_{(\gamma, d)}(u)=|p(u)|^{2}+|h a|^{2}, \quad N_{(\gamma, d)}(u)=|h a|^{4}\left|p^{\prime}(u)\right|^{2} .
$$

Then, by (3.3), (3.9), (6.1) and (6.4),

$$
\begin{aligned}
K d S & =\frac{2\left|F_{Z Z} F_{W}^{2}\right|^{2}+\cdots}{\left(\left|F_{Z}\right|^{2}+\left|F_{W}\right|^{2}\right)^{2}} \cdot \frac{1}{\left|F_{W}\right|^{2}} \cdot \frac{d Z \wedge d \bar{Z}}{-2 \sqrt{-1}} \\
& =\left\{\frac{2\left|p^{\prime}(u)\right|^{2}|h a|^{2}}{\left(|p(u)|^{2}+|h a|^{2}\right)^{2}}+o(W)\right\} \frac{d u \wedge d \bar{u}}{-2 \sqrt{-1}} .
\end{aligned}
$$

To compute the integrals in (2.19) using (6.5), we must know the number of sheets of $\mathcal{S}_{c} \cap H_{\text {orn }}^{(d)}\left(\gamma_{*} ; \rho ; \eta\right)$, lying over the $u$-plane, i.e. the number of values of $W$ when $u$ is given.

For this, in our situation let us first consider the special case where all $\gamma_{j}(y)$ are integral power series. In this case there is no conjugation,

$$
h:=O_{y}(f(\gamma(y), y))=O_{W}(F(0, W)) \text {, an integer. }
$$

When $u$ is given, there are $h$ distinct values of $W$ found by solving the equation

$$
c=a W^{h}+\cdots, \quad(c \text { is the value of the level: } F=c)
$$

where only those terms of order $>h$ depend on $u$. Hence the number of sheets is $h$.

Let $R>0$ be given, and fixed. Then

$$
\lim _{W \rightarrow 0} \int_{H_{\text {orn }}^{(d)}\left(\gamma_{*} ; R ; \eta\right) \cap \mathcal{S}_{c}} K d S=h \cdot \int_{|u| \leq R} \frac{2 b^{2}\left|p^{\prime}(u)\right|^{2}}{\left[b^{2}+|p(u)|^{2}\right]^{2}} \frac{d u \wedge d \bar{u}}{-2 \sqrt{-1}},
$$

where $b:=|h a|$. To compute the right-hand side, we write the polynomial $p(u)$ as

$$
p(u):=U(x, y)+\sqrt{-1} V(x, y), \quad u=x+\sqrt{-1} y \in \mathbb{C},
$$


where $U, V$ satisfy the Cauchy-Riemann equations. Using the latter we have

$$
\int \frac{2 b^{2}\left|p^{\prime}(u)\right|^{2}}{\left(b^{2}+|p(u)|^{2}\right)^{2}} \frac{d u \wedge d \bar{u}}{-2 \sqrt{-1}}=\int \frac{2 b^{2} d U \wedge d V}{\left(b^{2}+U^{2}+V^{2}\right)^{2}} \quad \text { (indefinite integrals). }
$$

Now $u \mapsto p(u)$ is a $\operatorname{deg} p(u)$-sheet branch covering map of $\mathbb{C}$. Letting $R \rightarrow \infty$,

$$
\begin{aligned}
\int_{\mathbb{C}} \frac{2 b^{2}\left|p^{\prime}(u)\right|^{2}}{\left(b^{2}+|p(u)|^{2}\right)^{2}} \frac{d u \wedge d \bar{u}}{-2 \sqrt{-1}} & =\operatorname{deg} p(u) \cdot \int_{\mathbb{R}^{2}} \frac{2 b^{2} d U \wedge d V}{\left(b^{2}+U^{2}+V^{2}\right)^{2}} \\
& =2 \pi \cdot \operatorname{deg} p(u)=2 \pi \cdot m\left(\mathcal{G C}\left(\gamma_{*}\right)\right) .
\end{aligned}
$$

On the other hand, by Lemma $\underline{3.2}(i)$, the following implication is true: for any $\alpha \in \mathbb{F}$,

$$
O_{y}(\alpha-\gamma) \geq d_{g r}\left(\gamma_{*}\right) \Longrightarrow O_{y}(f(\alpha(y), y))=h .
$$

As a consequence of this we have ( $\eta$ small enough):

$$
F(Z, W)=0,(Z, W) \in H_{\text {orn }}^{(d)}\left(\gamma_{*} ; R ; \eta\right) \Longrightarrow(Z, W)=0 .
$$

It follows that for $(Z, W) \in H_{\text {orn }}^{(d)}\left(\gamma_{*} ; R ; \eta\right) \cap \mathcal{S}_{c}$ the following conditions are equivalent:

$$
\text { (i) }(Z, W) \rightarrow 0, \quad(i i) c \rightarrow 0, \quad(\text { iii }) W \rightarrow 0 .
$$

Hence (for fixed $R$ )

$$
\lim _{W \rightarrow 0} \int_{H_{\text {orn }}^{(d)}\left(\gamma_{*} ; R ; \eta\right) \cap \mathcal{S}_{c}} K d S=\lim _{c \rightarrow 0} \int_{H_{o r n}^{(d)}\left(\gamma_{*} ; R ; \eta\right) \cap \mathcal{S}_{c}} K d S
$$

and this value is independent of $\eta$. Therefore

$$
\lim _{c \rightarrow 0} \int_{H_{o r n}^{(d)}\left(\gamma_{*} ; R ; \eta\right) \cap \mathcal{S}_{c}} K d S=\lim _{\eta \rightarrow 0} \lim _{c \rightarrow 0} \int_{H_{o r n}^{(d)}\left(\gamma_{*} ; R ; \eta\right) \cap \mathcal{S}_{c}} K d S=\mathscr{M}_{f}\left(\mathcal{D}^{(d)}\left(\gamma_{*} ; R\right)\right) .
$$

(The last equality is the definition (2.19) $)$ )

Thus, by (6.6), (6.7), we have

$$
\mathscr{M}_{f}\left(\mathcal{G C}\left(\gamma_{*}\right)\right)=\lim _{R \rightarrow \infty} \mathscr{M}_{f}\left(\mathcal{D}^{(d)}\left(\gamma_{*} ; R\right)\right)=h \cdot 2 \pi \cdot m\left(\mathcal{G C}\left(\gamma_{*}\right)\right) .
$$

Now, take any polar $\gamma_{j}$ such that $\gamma_{j *} \in \mathcal{G C}\left(\gamma_{*}\right)$. Then, by (6.8),

$$
O_{y}\left(f\left(\gamma_{j}(y), y\right)\right)=h \text {. }
$$

The number of such $\gamma_{j}$ is (by definition) $m\left(\mathcal{G C}\left(\gamma_{*}\right)\right)$. Hence, by (2.22), ([6.9),

$$
\mu_{f}\left(\mathcal{G C}\left(\gamma_{*}\right)\right)=m\left(\mathcal{G C}\left(\gamma_{*}\right)\right) \cdot(h-1) .
$$

It is then trivial to derive (2.24).

The general case, where $\gamma$ is fractional, can be derived from the above by the substitution $y \rightarrow y^{M}$, where $M$ is divisible by all $m_{\text {puiseux }}\left(\gamma_{j}\right)$, so that all $\gamma_{j}\left(y^{M}\right)$ are integral.

In this way $h$ is magnified to $M h$, and $\mathcal{S}_{c}$ is blown up to $M$ copies of itself. Let $R \rightarrow \infty$,

$$
\mathscr{M}_{f}\left(\mathcal{G C}\left(\gamma_{*}\right)\right)=\frac{1}{M} \cdot 2 \pi(M h) \cdot m\left(\mathcal{G C}\left(\gamma_{*}\right)\right)=2 \pi\left[\mu_{f}\left(\mathcal{G C}\left(\gamma_{*}\right)+m\left(\mathcal{G C}\left(\gamma_{*}\right)\right)\right] .\right.
$$

This completes the proof of Theorem C. 
Next we prove Theorem $D$.

Let a disc $D_{i s c}(0 ; \eta)$ be given (Definition2.8). Take constants $\rho, R, \rho>0$ sufficiently small, $R$ sufficiently large. We use $C_{\emptyset}, C_{\emptyset}^{\prime}$ to denote suitable non-zero constants; and

$$
A \approx B \text { means } 0<C_{\emptyset} \leq A / B \leq C_{\emptyset}^{\prime} .
$$

Let $0 \leq a<b \leq \infty$ be given. In this section we shall also use the short-hand

$$
H_{\text {orn }}(a, b):=\left\{\left.(Z, W) \in D_{i s c}(0 ; \eta)|R| W\right|^{b} \leq|Z| \leq \rho|W|^{a}\right\},
$$

with the convention that $R|W|^{\infty}=0$. If we set $Z=u W^{a}$, then

$$
(Z, W) \in H_{\text {orn }}(a, b) \Longleftrightarrow R|W|^{b-a} \leq|u| \leq \rho .
$$

Now, let us consider the Newton Polygon $\mathcal{N} \mathcal{P}(G)$ of a general $G(Z, W)$, with edges $\hat{E}_{i}$, and angles $\hat{\theta}_{i}, 0 \leq i \leq v$, where $\hat{E}_{0}$ is horizontal, $\hat{E}_{v}$ is vertical, $\hat{\theta}_{v}=\pi / 2$. We write

$$
\hat{e}_{i}:=\tan \hat{\theta}_{i}, \quad i=0, \ldots, v .
$$

For example, if $G=F$ in Fig[3, then $\hat{E}_{v-1}=E_{t o p}, \hat{E}_{v}$ is the vertical half-line at $(0, h)$.

The Vertex Lemma. Let $(k, q)$ be a given vertex of $\mathcal{N} \mathcal{P}(G)$, say the left vertex of $\hat{E}_{i}$ and the right vertex of $\hat{E}_{i+1}$, where $0 \leq i \leq v-1$. Then

$$
|G(Z, W)| \approx|Z|^{k}|W|^{q}, \quad(Z, W) \in H_{\text {orn }}\left(\hat{e}_{i}, \hat{e}_{i+1}\right) .
$$

In particular, for any given $\hat{e}, \hat{e}_{i} \leq \hat{e}<\hat{e}_{i+1}$, we have

$$
|G(Z, W)| \approx|Z|^{k}|W|^{q}, \quad(Z, W) \in H_{\text {orn }}\left(\hat{e}, \hat{e}_{i+1}\right) .
$$

Proof. Note that (6.11) is clearly true if $G$ is of the special form

$$
G(Z, W)=\text { unit } \cdot Z^{k} W^{q} \quad(|Z|,|W| \text { sufficiently small }) .
$$

For the general case, we can find a linear isomorphism, taking $\hat{E}_{i+1}$ to a vertical half-line and $\hat{E}_{i}$ to a horizontal one. Then $G(Z, W)$ is transformed to a function of the special form (6.12).

Corollary 6.1. If a dot $(P, Q)$ lies on or above $\mathcal{N P}(G)$, then $G$ "dominates" $Z^{P} W^{Q}$ in every horn domain. That is to say, for each $i, 0 \leq i \leq v-1$,

$$
|G(Z, W)|^{2}+\left|Z^{P} W^{Q}\right|^{2} \approx|G(Z, W)|^{2}, \quad(Z, W) \in H_{\text {orn }}\left(\hat{e}_{i}, \hat{e}_{i+1}\right) .
$$

(This does not mean " $\approx$ " is true for all $(Z, W)$. Because of $\rho$ and $R$, a number of "horn strips" are excluded.)

The Euler Lemma. Let $\omega:=\omega(e)$ be given. Consider the weighted Taylor expansion

$$
G(Z, W)=H_{D_{1}}^{(\omega)}(Z, W)+\cdots+H_{D_{k}}^{(\omega)}(Z, W)+\cdots, \quad D_{k}=\operatorname{deg}_{\omega} H_{D_{k}}^{(\omega)},
$$

where $H_{D}^{(\omega)}$ denotes a weighted homogeneous form of weighted degree $D$.

Then the weighted Taylor expansion of $W G_{W}+e Z G_{Z}$ is

$$
W G_{W}+e Z G_{Z}=D_{1} \cdot H_{D_{1}}^{(\omega)}(Z, W)+\cdots+D_{k} \cdot H_{D_{k}}^{(\omega)}(Z, W)+\cdots .
$$


In particular, $G$ and $W G_{W}+e Z G_{Z}$ have the same set of Newton dots,

$$
\mathcal{N} \mathcal{P}(G)=\mathcal{N} \mathcal{P}\left(W G_{W}+e Z G_{Z}\right)
$$

This lemma is an immediate consequence of Euler's Theorem (weighted version).

In the following corollary we take $G$ to be $F, 1<d_{g r}\left(\gamma_{*}\right)<\infty$. The edges and angles of $\mathcal{N P}(F)$ are $E_{i}$ and $\theta_{i}$, respectively, $0 \leq i \leq v$. We write

$$
e_{i}:=\tan \theta_{i}, \quad 0 \leq i \leq v . \quad\left(\tan \theta_{v}=\infty, E_{v-1}=E_{t o p} .\right)
$$

Corollary 6.2. Take any $e, e_{i} \leq e<e_{i+1}, 0 \leq i \leq v-1$. Then

$$
\frac{\left|W F_{W}\left(u W^{e}, W\right)\right|}{\left|W F_{W}\left(u W^{e}, W\right)+e u W^{e} F_{Z}\left(u W^{e}, W\right)\right|} \leq C_{\emptyset}, \quad R|W|^{e_{i+1}-e} \leq|u| \leq \rho .
$$

Proof. Let $(k, q)$ be the common vertex of $E_{i}, E_{i+1}$. Then, by the above two lemmas,

$$
\left|F\left(u W^{e}, W\right)\right| \approx\left|W F_{W}+e u W^{e} F_{Z}\right| \approx|u|^{k}|W|^{q+k e}
$$

Every dot of $W F_{W}$ lies on or above $\mathcal{N} \mathcal{P}(F)$. Hence

$$
\left|W F_{W}\left(u W^{e}, W\right)\right| \leq C_{\emptyset}^{\prime}|u|^{k}|W|^{q+k e}
$$

by Corollary6.1. This completes the proof.

Next consider $F_{Z}(Z, W)$, which has no Newton dot of the form $(0, q)$. We consider

$$
G(Z, W):=F_{Z}(Z, W)+W^{h-1}, \quad h \text { as in (3.4). }
$$

This time we denote the edges of $\mathcal{N} \mathcal{P}(G)$ by $E_{i}^{\prime}$, angles by $\theta_{i}^{\prime}, 0 \leq i \leq p+1$, and

$$
e_{i}^{\prime}:=\tan \theta_{i}^{\prime}, \quad 0 \leq i \leq p+1
$$

In particular,

$$
\tan \theta_{p}^{\prime}=d_{g r}\left(\gamma_{*}\right), \quad \tan \theta_{p+1}^{\prime}=\infty
$$

Lemma 6.3. The numbers $\left\{e_{i}\right\}$ in (6.15) and $\left\{e_{i}^{\prime}\right\}$ in (6.18) are related as follows:

$$
p \geq v-1 ; \quad e_{v-1}^{\prime} \geq e_{v-1} ; \quad e_{i}^{\prime}=e_{i}, 0 \leq i \leq v-2(\text { if } v \geq 2)
$$

where $e_{v-1}^{\prime}>e_{v-1}$ if and only if $\left(\widehat{m}_{t o p}, \widehat{q}_{t o p}\right)=\left(m_{\text {top }}, q_{\text {top }}\right)$ (see Fig. 4).

This relationship can be seen clearly in Fig.4. Note that $(k, q), k>0$, is a Newton dot of $F$ if, and only if, it is one of $Z F_{Z}$.

Corollary 6.4. Given $i, 0 \leq i \leq p-1, F_{Z}$ “dominates" $F_{W}$ in $H_{\text {orn }}\left(e_{i}^{\prime}, e_{i+1}^{\prime}\right)$. That is,

$$
\left|F_{Z}\right|^{2}+\left|F_{W}\right|^{2} \approx\left|F_{Z}\right|^{2}, \quad(Z, W) \in H_{\text {orn }}\left(e_{i}^{\prime}, e_{i+1}^{\prime}\right) .
$$

However, $F_{Z}$ "is dominated by" $F_{W}$ in $H_{\text {orn }}\left(e_{p}^{\prime}, e_{p+1}^{\prime}\right)$. That is,

$$
\left|F_{Z}\right|^{2}+\left|F_{W}\right|^{2} \approx\left|F_{W}\right|^{2}, \quad(Z, W) \in H_{o r n}\left(e_{p}^{\prime}, e_{p+1}^{\prime}\right)
$$


Proof. All dots of $F_{W}$ lie on or above $\mathcal{N P}(G), G$ defined in (6.17). On the other hand,

$$
\left|F_{Z}(Z, W)\right| \approx|G(Z, W)| \quad(Z, W) \in H_{\text {orn }}\left(e_{i}^{\prime}, e_{i+1}^{\prime}\right)
$$

where $0 \leq i \leq p-1$. Hence (6.19) follows from Corollary 6.1.

Because $(0, h-1)$ is a vertex of $\mathcal{N P}(G),(6.20)$ is a consequence of the Vertex Lemma and Corollary 6.1.

We now establish four lemmas, Lemma 6.5 to Lemma6.8, then derive Theorem D.

Lemma 6.5. Let $\gamma_{*}$ be a given polar, $1<d_{g r}\left(\gamma_{*}\right)<\infty$. Take $e^{\prime}, 1<e^{\prime}<d_{g r}\left(\gamma_{*}\right)$.

Take $i$ such that $e_{i-1}^{\prime} \leq e^{\prime}<e_{i}^{\prime}$ (hence $\left.i \leq p\right)$. Let $\epsilon>0$ be sufficiently small. Then

$$
\mathscr{M}_{f}\left(\mathcal{D}^{\left(e^{\prime}\right)}\left(\gamma_{*}, \epsilon\right)\right)=\mathscr{M}_{f}\left(\mathcal{L}^{\left(e_{i}^{\prime}\right)}\left(\gamma_{*}\right)\right)
$$

where $\mathcal{D}, \mathcal{L}$ are defined in (2.6), 2.7). In particular,

$$
e_{p-1}^{\prime} \leq e^{\prime}<d_{g r}\left(\gamma_{*}\right)\left(\text { and } e^{\prime}>1\right) \Longrightarrow \mathscr{M}_{f}\left(\mathcal{D}^{\left(e^{\prime}\right)}\left(\gamma_{*}, \epsilon\right)\right)=\mathscr{M}_{f}\left(\mathcal{G C}\left(\gamma_{*}\right)\right) \text {. }
$$

Lemma 6.6. Let $\mathcal{D}^{(e)}\left(\alpha_{*}, \epsilon\right)$ be a given infinitesimal disc, $1<e<\infty, \epsilon>0$.

Suppose this disc is disjoint from every minimal gradient canyon (see (2.25)). Then

$$
\mathscr{M}_{f}\left(\mathcal{D}^{(e)}\left(\alpha_{*}, \epsilon\right)\right)=0
$$

(The disc may contain singleton canyons.)

On the other hand, if there is a polar $\gamma_{*}$ such that

$$
\mathcal{D}^{(e)}\left(\alpha_{*}, \epsilon\right) \subset \mathcal{G C}\left(\gamma_{*}\right), \quad e>d_{g r}\left(\gamma_{*}\right)>1,
$$

then

$$
\mathscr{M}_{f}\left(\mathcal{L}^{(e)}\left(\alpha_{*}\right)\right)=0
$$

We now prove Lemma 6.5.

We show the difference of the two sides of (6.21) is zero. With the substitution $Z=u W^{e^{\prime}}$, this amounts to showing that

$$
\lim _{R \rightarrow \infty} \lim _{W \rightarrow 0} \int_{R|W|^{\delta_{i}} \leq|u| \leq \epsilon} K d S=0, \quad \delta_{i}:=e_{i}^{\prime}-e^{\prime}, \epsilon>0 \text { a small constant. }
$$

Now, like (6.2), we have

$$
d Z=Q W^{e^{\prime}} d u, \quad Q:=\frac{W F_{W}}{W F_{W}+e^{\prime} u F_{Z}},
$$

where $|Q| \leq C_{\emptyset}$ by $(\underline{6.16})$. Hence

$$
K d S=\frac{2\left|\Delta_{F}+\gamma^{\prime \prime} F_{Z}^{3}\right|^{2}}{\left(\left|F_{Z}\right|^{2}+\left|F_{W}\right|^{2}\right)^{2}} \cdot \frac{|Q|^{2}}{\left|F_{W}\right|^{2}} \cdot|W|^{2 e^{\prime}} \cdot \frac{d u \wedge d \bar{u}}{-2 \sqrt{-1}} .
$$

We divide the proof into two cases:

$$
\text { (I) } \tan \theta_{1}^{\prime}=\tan \theta_{1}, \quad \text { (II) } \tan \theta_{1}^{\prime}>\tan \theta_{1} \text {. }
$$

Let us first consider (I). This is the only difficult case.

In the first place, since $d_{g r}\left(\gamma_{*}\right)>1$, we know $p \geq 2$. (See Fig.4.) 
Consider the weight system $\omega\left(e^{\prime}\right)$ (where $e_{i-1}^{\prime} \leq e^{\prime}<e_{i}^{\prime}$ ). Let us write

$$
\mathcal{I}_{\omega\left(e^{\prime}\right)}\left(F_{Z}\right)(Z, W):=c_{k} Z^{k} W^{q}+c_{k+1} Z^{k+1} W^{q-e^{\prime}}+\cdots, \quad c_{k} \neq 0
$$

where $k \geq 1$, since $\gamma$ is a polar. If $e^{\prime} \geq e_{1}^{\prime}$, then $q \geq 1$.

Note that $(k, q)$ is the common vertex of $E_{i-1}^{\prime}$ and $E_{i}^{\prime}$, and

$$
e_{i-1}^{\prime}<e^{\prime}<e_{i}^{\prime} \Longrightarrow \mathcal{I}_{\omega\left(e^{\prime}\right)}\left(F_{Z}\right)(Z, W)=c_{k} Z^{k} W^{q} \text {, a monomial. }
$$

To compute (6.25), we use the expansion (3.7). We define $K_{i}, 1 \leq i \leq 4$, and show

$$
\lim _{R \rightarrow \infty} \lim _{W \rightarrow 0} \int_{R|W|^{\delta_{i} \leq|u| \leq \epsilon}} K_{i} \cdot \frac{d u \wedge d \bar{u}}{-2 \sqrt{-1}}=0, \quad 1 \leq i \leq 4 .
$$

It will then be obvious that (6.25) is true (an easy use of the triangle inequality).

To begin, we define

$$
K_{1}:=\frac{\left|F_{Z Z} F_{W}^{2}\right|^{2}}{\left(\left|F_{Z}\right|^{2}+\left|F_{W}\right|^{2}\right)^{2}} \cdot \frac{|Q|^{2}}{\left|F_{W}\right|^{2}} \cdot|W|^{2 e^{\prime}} .
$$

Using the fact that $F_{Z}$ dominates $F_{W}$ (Corollary6.4), we have

$$
K_{1} \leq C_{\emptyset} \frac{\left|Z F_{Z Z}\right|^{2}}{\left|F_{Z}\right|^{2}} \frac{\left|F_{W}\right|^{2}}{\left|F_{Z}\right|^{2}} \cdot \frac{|W|^{2 e^{\prime}}}{|Z|^{2}}, \quad R|W|^{\delta_{i}} \leq|u| \leq \epsilon .
$$

Then, since $Z F_{Z Z}$ and $F_{Z}$ have the same Newton dots,

$$
K_{1} \leq C_{\emptyset}^{\prime} \frac{\left|F_{W}\right|^{2}}{\left|F_{Z}\right|^{2}} \cdot \frac{|W|^{2 e^{\prime}}}{|Z|^{2}}, \quad R|W|^{\delta_{i}} \leq|u| \leq \epsilon .
$$

Now let us first consider the case $e_{p-1}^{\prime} \leq e^{\prime}<d_{g r}\left(\gamma_{*}\right)$.

Applying the Vertex Lemma to the vertex $(k, q)$, we have

$$
\left|F_{Z}\right| \approx|u|^{k}|W|^{q+k e^{\prime}}, \quad R|W|^{\delta_{p}} \leq|u| \leq \epsilon .
$$

Since $(0, h-1)$ is the only $\operatorname{dot}$ of $F_{W}$ on $L^{*}$, we also have

$$
\left|F_{W}\right| \approx|W|^{h-1}, \quad h-1=q+k d_{g r}\left(\gamma_{*}\right) . \quad(\text { See Fig, 4, })
$$

It follows that

$$
K_{1} \leq C_{\emptyset}^{\prime} \frac{\left|F_{W}\right|^{2}}{\left|F_{Z}\right|^{2}} \cdot \frac{|W|^{2 e^{\prime}}}{|u|^{2}|W|^{2 e^{\prime}}} \leq C_{\emptyset}^{\prime \prime} \frac{|W|^{2 k \delta_{p}}}{|u|^{2(k+1)}}, \quad R|W|^{\delta_{p}} \leq|u| \leq \epsilon .
$$

We use polar coordinates in the following computation:

$$
\int_{R|W|^{\delta_{p}} \leq|u| \leq \epsilon} K_{1} \frac{d u \wedge d \bar{u}}{-2 \sqrt{-1}} \leq C_{\emptyset} \int_{R|W|^{\delta_{p}}}^{\epsilon} \frac{|W|^{2 k \delta_{p}} \cdot r d r}{r^{2(k+1)}}=C_{\emptyset}\left[\frac{1}{R^{2 k}}-\frac{|W|^{2 k \delta_{p}}}{\epsilon^{2 k}}\right],
$$

where $u=r \exp \sqrt{-1} \theta$. Thus (with $\epsilon>0$ fixed)

$$
\lim _{R \rightarrow \infty} \lim _{W \rightarrow 0} \int_{R|W|^{\delta_{p}} \leq|u| \leq \epsilon} K_{1} \cdot \frac{d u \wedge d \bar{u}}{-2 \sqrt{-1}}=0 .
$$

Next suppose $e_{i-1}^{\prime} \leq e^{\prime}<e_{i}^{\prime}$, where $2 \leq i \leq p-1$. 
As pointed earlier, $k \geq 1, q \geq 1$. Hence

$$
\frac{1}{q} W \frac{\partial}{\partial W}\left(Z^{k} W^{q}\right)=\frac{1}{k} Z \frac{\partial}{\partial Z}\left(Z^{k} W^{q}\right)=Z^{k} W^{q} .
$$

It follows that

Then, by (6.29),

$$
\left|W F_{W}\right| \approx\left|Z F_{Z}\right|, \quad R|W|^{\delta_{i}} \leq|u| \leq \epsilon .
$$

$$
K_{1} \leq C_{\emptyset}|W|^{2\left(e^{\prime}-1\right)}, \quad \lim _{W \rightarrow 0} \int_{R|W| \delta_{i} \leq|u| \leq \epsilon} K_{1} \frac{d u \wedge d \bar{u}}{-2 \sqrt{-1}}=0 .
$$

The equality is independent of $R$. Letting $R \rightarrow \infty$, the limit is of course still zero.

Now we define $K_{2}, K_{3}$, where $e_{i-1}^{\prime} \leq e^{\prime} \leq e_{i}^{\prime}, 2 \leq i \leq p$,

$$
K_{2}:=\frac{\left|F_{Z W} F_{Z} F_{W}\right|^{2}|Q|^{2}}{\left(\left|F_{Z}\right|^{2}+\left|F_{W}\right|^{2}\right)^{2}} \cdot \frac{|W|^{2 e^{\prime}}}{\left|F_{W}\right|^{2}}, \quad K_{3}:=\frac{\left|F_{W W} F_{Z}^{2}\right|^{2}|Q|^{2}}{\left(\left|F_{Z}\right|^{2}+\left|F_{W}\right|^{2}\right)^{2}} \cdot \frac{|W|^{2 e^{\prime}}}{\left|F_{W}\right|^{2}} .
$$

Since $F_{Z}$ dominates $F_{W}$ in the horn domain $R|W|^{\delta_{i}} \leq|u| \leq \epsilon$, we have

$$
K_{2} \leq C_{\emptyset} \frac{\left|W F_{Z W}\right|^{2}}{\left|F_{Z}\right|^{2}}|W|^{2\left(e^{\prime}-1\right)}, \quad K_{3} \leq C_{\emptyset} \frac{\left|W F_{W W}\right|^{2}}{\left|F_{W}\right|^{2}}|W|^{2\left(e^{\prime}-1\right)}
$$

It follows that

$$
K_{2} \leq C_{\emptyset}^{\prime}|W|^{2\left(e^{\prime}-1\right)}, \quad K_{3} \leq C_{\emptyset}^{\prime}|W|^{2\left(e^{\prime}-1\right)},
$$

where $e^{\prime}-1>0$. Hence

$$
\lim _{W \rightarrow 0} \int_{R|W|^{\delta_{i}} \leq|u| \leq \epsilon} K_{2} \frac{d u \wedge d \bar{u}}{-2 \sqrt{-1}}=0, \quad \lim _{W \rightarrow 0} \int_{\left.R|W|\right|^{\delta_{i}} \leq|u| \leq \epsilon} K_{3} \frac{d u \wedge d \bar{u}}{-2 \sqrt{-1}}=0 .
$$

Again, the values are independent of $R$. Letting $R \rightarrow \infty$, the limits are still zero.

Finally, we consider $K_{4}, e_{i-1}^{\prime} \leq e^{\prime} \leq e_{i}^{\prime}, 2 \leq i \leq p$, where

$$
K_{4}:=\frac{\left|\gamma^{\prime \prime} F_{Z}^{3}\right|^{2}|Q|^{2}}{\left(\left|F_{Z}\right|^{2}+\left|F_{W}\right|^{2}\right)^{2}} \cdot \frac{|W|^{2 e^{\prime}}}{\left|F_{W}\right|^{2}} \leq C_{\emptyset} \frac{\left|Z F_{Z}\right|^{2}}{\left|W F_{W}\right|^{2}} \cdot \frac{\left|\gamma^{\prime \prime}\right|^{2}|W|^{2\left(e^{\prime}+1\right)}}{|Z|^{2}} .
$$

We have

$$
K_{4} \leq C_{\emptyset}^{\prime} \frac{|W|^{2\left[1+O\left(\gamma^{\prime \prime}\right)\right]}}{|u|^{2}}, \quad \lim _{W \rightarrow 0} \int_{R|W|^{\delta_{i} \leq|u| \leq \epsilon}} K_{4} \frac{d u \wedge d \bar{u}}{-2 \sqrt{-1}}=0,
$$

the last equation follows from an elementary result in Calculus:

$$
\lim _{W \rightarrow 0}|W|^{\nu} \cdot \ln |W|=0, \quad \nu:=1+O\left(\gamma^{\prime \prime}\right)>0 .
$$

By (6.26), the above results obviously imply (6.25).

To complete the proof of (I) it remains to consider the case $1<e^{\prime}<e_{1}^{\prime}=e_{1}$.

In this case, there is no need to change variable (3.1), (6.28) reduces to

$$
\mathcal{I}_{\omega\left(e^{\prime}\right)}(f)(z, w)=c_{m-1} z^{m-1},
$$

and then $f_{z}$ dominates $f_{w}, z f_{z}$ dominates $w f_{w}$, etc.. It follows that

$$
K_{j} \leq C_{\emptyset}|w|^{2\left(e^{\prime}-1\right)}, 1 \leq j \leq 3, \quad R|W|^{\delta_{1}} \leq|u| \leq \epsilon,
$$


while $K_{4}$ need not be considered. Since $e^{\prime}>1$, we have

$$
\lim _{w \rightarrow 0} \int_{R|w|^{\delta_{1}} \leq|u|<\epsilon} K_{j} \frac{d u \wedge d \bar{u}}{-2 \sqrt{-1}}=0, \quad 1 \leq j \leq 3 .
$$

Again this implies (6.25).

To complete the proof of Lemma 6.5 it remains to consider the case (II) in (6.27).

By Theorem 2.1 in [7], (II) can happen only if $f(z, w)$ has weighted Taylor expansion

$$
f(z, w)=\left(C_{\emptyset} z^{m}+C_{\emptyset}^{\prime} w^{n}\right)+\cdots, \quad \omega(z):=n / m=e_{1} \geq 1, \omega(w)=1,
$$

where "..." means "higher weighted order terms".

In this case $f_{z}$ dominates $f_{w}$. The proof is the same as above.

Next we prove Lemma 6.6.

Although we do not assume $\epsilon>0$ is sufficiently small, we can add this assumption since our horn domains are compact.

To show (6.23), let us first consider the case where the disc contains a singleton canyon $\mathcal{G C}\left(\gamma_{*}\right)$. Since $\epsilon>0$ is sufficiently small, we have $J^{(e)}\left(\gamma_{*}\right)=J^{(e)}\left(\alpha_{*}\right)$.

Since $d_{g r}\left(\gamma_{*}\right)=\infty, \gamma$ is a multiple root of $f(z, w)$, say of multiplicity $k, k \geq 2$. Then $Z$ is a multiple factor of $F(Z, W)$ of the same multiplicity.

Consider $\mathcal{N} \mathcal{P}(F)$, with edges $E_{0}, \ldots, E_{v}$. The vertical edge $E_{v}$ sits on $(k, q)$, for some $q$.

We must have $e \geq \tan \theta_{v-1}$, for otherwise, as a consequence of Theorem 2.1 in [7] (or Lemma 3.3 in [6]), there would exist a polar $\hat{\gamma}$ such that

$$
O_{y}(\gamma(y)-\hat{\gamma}(y))=\tan \theta_{v-1}>e, \quad f(\hat{\gamma}(y), y) \not \equiv 0 .
$$

There would then be a contradiction:

$$
d_{g r}\left(\hat{\gamma}_{*}\right)<\infty, \quad \mathcal{G C}\left(\hat{\gamma}_{*}\right) \subset \mathcal{D}^{(e)}\left(\alpha_{*} ; \epsilon\right) .
$$

We can therefore restrict our attention to $H_{\text {orn }}\left(\tan \theta_{v-1}, \infty\right)$. In this horn domain $F_{Z}$ dominates $F_{W}$. The argument for proving Lemma 6.5 can be applied to prove (6.23).

We can now assume the disc is also disjoint from singleton canyons.

Consider $G:=f(Z+\alpha(W), W)$ and $\mathcal{N} \mathcal{P}(G)$. By the above assumption, $G$ must have a dot on $Z=0$ or on $Z=1$, for otherwise $\alpha$ would be a multiple root of $f$, a contradiction.

In either cases, the same argument for proving Lemma 6.5 can be applied to prove 6.23 .

For (6.24), we set $Z=u W^{d+\delta}, d:=d_{g r}\left(\gamma_{*}\right), \delta:=e-d$, and get $K d S \approx|W|^{2 \delta} d u \wedge d \bar{u}$, whence (6.24). This completes the proof of Lemma 6.6.

Next we introduce two more lemmas.

With the substitution $z=u w$, we have, as before,

$$
K d S=\frac{2\left|\Delta_{f}\right|^{2}}{\left(\left|f_{z}\right|^{2}+\left|f_{w}\right|^{2}\right)^{2}} \cdot \frac{|w|^{4}}{\left|w f_{w}+u w f_{z}\right|^{2}} \cdot \frac{d u \wedge d \bar{u}}{-2 \sqrt{-1}}
$$


Lemma 6.7. Take a multiple root $z_{i}$ of $H_{m}(z, w)$ in (2.9), i.e. $m_{i} \geq 2$. Then

$$
\lim _{\epsilon \rightarrow 0} \mathscr{M}_{f}\left(\mathcal{D}^{(1)}\left(z_{i *} ; \epsilon\right)\right)=\mathscr{M}_{f}\left(\mathcal{L}^{(1+\delta)}\left(z_{i *}\right)\right)
$$

where $\delta>0$ is sufficiently small.

If $z_{i}$ is a simple root, i.e. $m_{i}=1$, then

$$
\lim _{\epsilon \rightarrow 0} \mathscr{M}_{f}\left(\mathcal{D}^{(1)}\left(z_{i *} ; \epsilon\right)\right)=0 .
$$

Proof. For simplicity, we write $H(z, w):=H_{m}(z, w)$. We can assume $z_{i}=0$.

If 0 is a simple root of $H$, then it is not a common root of $H_{z}, H_{w}$. Hence

$$
\left|f_{z}\right|^{2}+\left|f_{w}\right|^{2} \approx\left|H_{z}\right|^{2}+\left|H_{w}\right|^{2} \approx C_{\emptyset}|w|^{2(m-1)} \quad \text { near } u=0 .
$$

We also have

$$
\left|\Delta_{f}\right|^{2} \leq C_{\emptyset}|w|^{6 m-8} \cdot|u|^{2}, \quad\left|w f_{w}+u w f_{z}\right|^{2} \approx|w|^{2 m} \cdot|u|^{2},
$$

where the last relation follows from Euler's Theorem. Hence we have

$$
|K d S| \leq C_{\emptyset}^{\prime}, \quad \lim _{\epsilon \rightarrow 0} \int_{|u| \leq \epsilon} K d S=0 .
$$

If 0 is a multiple root of $H$, say of multiplicity $k+1$, it is a common root of $H_{z}, H_{w}$, of multiplicity $k$, and vice versa.

In this case, $H_{z}$ dominates $H_{w}$ in the horn domain $H_{\text {orn }}(1,1+\delta)$. We can repeat part of the proof of Lemma6.5 to complete the proof.

Lemma 6.8. Consider the "punched" plane

$$
\mathcal{P}(\epsilon):=\mathbb{C}_{\text {enriched }}-\bigcup_{i=1}^{r} \mathcal{D}^{(1)}\left(z_{i *} ; \epsilon\right), \quad \epsilon>0 .
$$

We have

$$
\lim _{\epsilon \rightarrow 0} \mathscr{M}_{f}(\mathcal{P}(\epsilon))=2 \pi m(r-1) .
$$

Proof. Let us first assume $r=1$. Then we can assume

$$
H(z, w):=H_{m}(z, w)=z^{m} .
$$

By Euler's Theorem,

$$
w f_{w}+u w f_{z}=m H(u w, w)+\cdots=m u^{m} w^{m}+\cdots .
$$

Of course, we also have

$$
O\left(f_{z}\right)=m-1<O\left(f_{w}\right), \quad O\left(f_{z z}\right)=m-2<O\left(f_{z w}\right), \text { etc. }
$$

It follows that

$$
O\left(\Delta_{f}\right)>3 m-4, \quad K d S=o(w) \cdot \frac{d u \wedge d \bar{u}}{-2 \sqrt{-1}},
$$

and hence, for any $a>\epsilon$,

$$
\lim _{w \rightarrow 0} \int_{\epsilon \leq|u| \leq a} K d S=0 .
$$

Therefore (6.33) is true in the case $r=1$. 
Next we assume $r \geq 2$. In this case, the initial form of $\Delta_{f}$ is

$$
\Delta_{H}=\left|\begin{array}{ccc}
H_{z z} & H_{z w} & H_{z} \\
H_{w z} & H_{w w} & H_{w} \\
H_{z} & H_{w} & 0
\end{array}\right| .
$$

This can be proved as follows. Since $r \geq 2$, we can assume

$$
H(z, w)=c_{k} z^{k} w^{m-k}+\cdots+c_{m} z^{m}, \quad c_{k} \neq 0 \neq c_{m}, 0<k<m .
$$

Then, by a simple computation, we have

$$
\Delta_{H}=c_{k}^{3} m k(m-k) z^{3 k-2} w^{3 q-2}+\cdots \not \equiv 0,
$$

whence $\Delta_{H}$ is the initial form of $\Delta_{f}$.

(It is not difficult to see that $\Delta_{H} \equiv 0$ if and only if $H$ has only one factor, i.e. $r=1$.)

We also have

$$
w f_{w}+u w f_{z}=m H(u w, w)+\cdots=m H(u, 1) w^{m}+\cdots .
$$

Hence we can rewrite (6.32) in the form

$$
K d S=\left\{\frac{2\left|\mathcal{R}^{\prime}(u)\right|^{2}}{\left(1+|\mathcal{R}(u)|^{2}\right)^{2}}+o(W)\right\} \frac{d u \wedge d \bar{u}}{-2 i}, \quad \mathcal{R}(u):=\frac{H_{z}(u, 1)}{H_{w}(u, 1)} .
$$

If $\left(z-z_{i} w\right)^{m_{i}}, m_{i} \geq 2$, is a factor of $H(z, w)$, then $\left(z-z_{i} w\right)^{m_{i}-1}$ is a common factor of $H_{z}, H_{w}$, and vice versa. Hence, having canceled all common factors, we have

$$
\mathcal{R}(u)=p(u) / q(u), \quad \operatorname{deg} p(u)=r-1 \geq \operatorname{deg} q(u),
$$

where $p(u), q(u)$ are relatively prime.

Now, the rational function

$$
\mathcal{R}: \mathbb{C} \longrightarrow \mathbb{C}, \quad u \mapsto \mathcal{R}(u):=U+i V,
$$

is an $(r-1)$-fold branch covering, where $U, V$ satisfy the Cauchy-Riemann equations.

Take $\delta>0$. Consider the punched disk:

$$
P(\delta):=\left\{(z, w) \in D_{i s c}(0 ; \eta)|| z-z_{i} w|\geq \delta| w \mid, 1 \leq i \leq r\right\} .
$$

An important observation is that the surface $\mathcal{S}_{c} \cap P(\delta)$ consists of $m$ sheets, since for each generic $u$ the surface has $m$ distinct intersecting points with the line $z=u w$.

When the integral of $K$ on $\mathcal{S}_{c} \cap P(\delta)$ is transformed to one over the complex $(U+i V)$-plane, the latter ought to be multiplied by a factor of $m(r-1)$. Thus

$$
\lim _{\delta \rightarrow 0} \lim _{W \rightarrow 0} \int_{\mathcal{S}_{c} \cap P(\delta)} K d S=m(r-1) \cdot \lim _{\delta \rightarrow 0} \int_{\mathbb{C}(\delta)} \frac{2 d U \wedge d V}{\left[1+U^{2}+V^{2}\right]^{2}}=2 \pi m(r-1),
$$

where $\mathbb{C}(\delta):=\left\{z|| z-z_{i} \mid \geq \delta, 1 \leq i \leq r\right\}$. This completes the proof of Lemma 6.8 , 
We can now complete the proof of Theorem $D$.

Let $\mathcal{L}^{(e)}, e>1$, be a given infinitesimal line.

If $\mathcal{L}^{(e)}$ does not contain any polar $\gamma_{*}$, then $\mathscr{M}_{f}\left(\mathcal{L}^{(e)}\right)=0$ by Lemma (6.6).

Otherwise, we can permute the indices, if necessary, so that $\left\{\mathcal{G C}\left(\gamma_{1 *}\right), \ldots, \mathcal{G C}\left(\gamma_{l *}\right)\right\}$ are the (distinct) gradient canyons contained in $\mathcal{L}^{(e)}$.

It can happen that some $\gamma_{j *}$ has $d_{g r}\left(\gamma_{j *}\right)=e$. Then, by Theorem $\mathrm{B}, \mathcal{G C}\left(\gamma_{j *}\right)$ is necessarily the only canyon contained in $\mathcal{L}^{(e)}$; so that $l=1, \mathcal{L}^{(e)}=\mathcal{G C}\left(\gamma_{1 *}\right)$ is minimal, (2.26) holds.

Now suppose

$$
e<d_{g r}\left(\gamma_{j *}\right) \leq \infty, \quad 1 \leq j \leq l
$$

In this case,

$$
\mathscr{M}_{f}\left(\mathcal{L}^{(e)}\right)=\sum_{j=1}^{l} \mathscr{M}_{f}\left(\mathcal{G C}\left(\gamma_{j *}\right)\right),
$$

where singleton canyons can be discarded. That is, (2.26) is true.

A proof of (6.35) is as follows. Take $\alpha_{*} \in \mathcal{L}^{(e)}$, which is not in one of the above canyons. Let $\alpha$ be a coordinate of $\alpha_{*}$. Take $k$ such that

$$
O\left(\alpha-\gamma_{k}\right)=\max \left\{O\left(\alpha-\gamma_{j}\right) \mid 1 \leq j \leq l\right\} .
$$

Now consider

$$
Q:=\left(q_{1}, q_{2}, q_{3}, q_{4}\right) \in \mathbb{Q}^{4}, \quad q_{1}:=O\left(\alpha-\gamma_{k}\right), q_{2}>0,
$$

and let

$$
\gamma^{(Q)}(y):=\gamma_{k}(y)+\left(q_{3}+\sqrt{-1} q_{4}\right) y^{q_{1}} .
$$

If the rational numbers $q_{2}, q_{3} q_{4}$ are properly chosen, then

$$
\alpha_{*} \in \mathcal{D}^{\left(q_{1}\right)}\left(\gamma_{*}^{(Q)} ; q_{2}\right), \quad \mathcal{D}^{\left(q_{1}\right)}\left(\gamma_{*}^{(Q)} ; q_{2}\right) \cap\left[\cup_{1 \leq j \leq l} \mathcal{G C}\left(\gamma_{j *}\right)\right]=\emptyset .
$$

Of course there are uncountably many $\alpha_{*}$, but the number of discs appearing in (6.36) is countable. We can list them as $\mathcal{D}_{n}, n \in \mathbb{Z}^{+}$.

Each $\mathcal{D}_{n}$ is disjoint from the minimal gradient canyons, so by Lemma6.6,

$$
\mathscr{M}_{f}\left(\mathcal{D}_{n}\right)=0, \quad 1 \leq n<\infty,
$$

and then (6.35) follows from the identity

$$
\mathcal{L}^{(e)}=\left[\cup_{1 \leq j \leq l} \mathcal{G C}\left(\gamma_{j *}\right)\right] \cup\left[\cup_{1 \leq n<\infty} \mathcal{D}_{n}\right] .
$$

Finally, to prove (2.27), let $\gamma(y)=c y+\cdots$ be given. By Lemma 3.2, $1<d_{g r}\left(\gamma_{*}\right) \leq \infty$ if and only if $c$ is a multiple root of $H_{m}(x, 1)=0$. Hence (2.27) follows from Lemma 6.7 and Lemma 6.8 and the previous calculation (6.35)

Remark 6.9. The above lemmas can be used to show that $\mathscr{M}_{f}$ (defined on the enriched discs of positive radius) satisfies the hypothesis of Carathéodory's Extension Theorem, hence extends to a measure on the $\sigma$-algebra generated by the discs. 


\section{Proof of Theorem E}

Let us write (2.21) as

$$
\mu_{f}=\sum_{j=1}^{m-1} \mu_{f}\left(\gamma_{j}\right), \quad \mu_{f}\left(\gamma_{j}\right):=O_{y}\left(f\left(\gamma_{j}(y), y\right)\right)-1 .
$$

We shall compute each $\mu_{f}\left(\gamma_{j}\right)$.

For convenience, let us re-name and list the roots of $f_{z}^{(\epsilon)}(z, w)$ and $f_{z}^{(\delta)}(z, w)$ as

$$
\left\{\gamma_{1}^{(\epsilon)}, \ldots, \gamma_{m-1}^{(\epsilon)}\right\} \text { and }\left\{\gamma_{1}^{(\delta)}, \ldots, \gamma_{m-1}^{(\delta)}\right\}
$$

respectively, in such a way that

$$
d_{g r}\left(\gamma_{j}\right)=O\left(\gamma_{j}-\gamma_{j}^{(\epsilon)}\right)=O\left(\gamma_{j}-\gamma_{j}^{(\delta)}\right)=O\left(\gamma_{j}^{(\epsilon)}-\gamma_{j}^{(\delta)}\right), \quad 1 \leq j \leq m-1 .
$$

It is easy to see that

$$
O\left(\gamma_{j}-\gamma_{i}\right) \geq d_{g r}\left(\gamma_{j}\right) \Longrightarrow O\left(\gamma_{j}^{(\epsilon)}-\gamma_{i}\right)=d_{g r}\left(\gamma_{j}\right),
$$

and

$$
O\left(\gamma_{j}-\gamma_{i}\right)<d_{g r}\left(\gamma_{j}\right) \Longrightarrow O\left(\gamma_{j}^{(\epsilon)}-\gamma_{i}\right)=O\left(\gamma_{j}-\gamma_{i}\right) .
$$

Now consider $\mathcal{N} \mathcal{P}(F), F(Z, W):=f\left(Z+\gamma_{j}(W), W\right)$, as in Fig. 3. Recall that $(i, q), i \geq 1$, is a dot of $F$ if and only if $(i-1, q)$ is one of $F_{Z}$.

The edges of $\mathcal{N} \mathcal{P}\left(F_{Z}\right)$ are denoted by $E_{i}^{\prime}$ with co-slopes $e_{i}^{\prime}$ as in (6.18), $0 \leq i \leq p+1$, where $E_{p+1}^{\prime}$ is vertical, $\left(m_{i}^{\prime}, q_{i}^{\prime}\right)$ is the right vertex of $E_{i}^{\prime}$.

Amongst the dots of $F_{Z}$ on $L^{*}$, let $\left(m_{*}, q_{*}\right)$ be the lowest one, i.e., $q_{*}$ is minimal. (As shown in Fig, $F$ has no dots on $L$ lying below $\left(m_{*}+1, q_{*}\right)$.) By Theorem 2.1 in [7],

$$
\sharp\left\{i \mid O\left(\gamma_{j}-\gamma_{i}\right) \geq d_{g r}\left(\gamma_{j}\right)\right\}=m_{*} .
$$

To compute $\mu_{f}\left(\gamma_{j}\right)$, let us first assume $d_{g r}\left(\gamma_{j}\right)>1$.

Of course $\left(m_{*}, q_{*}\right)$ is a vertex of $\mathcal{N} \mathcal{P}\left(F_{Z}\right)$, say

$$
\left(m_{*}, q_{*}\right)=\left(m_{k+1}^{\prime}, q_{k+1}^{\prime}\right), \quad k \leq p .
$$

Take any $l, 1 \leq l \leq k$. By the same theorem in [7],

$$
\#\left\{i \mid O\left(\gamma_{j}-\gamma_{i}\right)=e_{l}^{\prime}\right\}=m_{l}^{\prime}-m_{l+1}^{\prime} .
$$

It follows that (see Fig. 30)

$$
h-1=m_{k+1}^{\prime} d_{g r}\left(\gamma_{j}\right)+\sum_{l=1}^{k}\left(m_{l}^{\prime}-m_{l+1}^{\prime}\right) e_{l}^{\prime} .
$$

By (7.2), (7.3)

$$
m_{k+1}^{\prime}=\sharp\left\{i \mid O_{y}\left(\gamma_{j}^{(\epsilon)}-\gamma_{i}^{(\delta)}\right)=d_{g r}\left(\gamma_{j}\right)\right\}, \quad m_{l}^{\prime}-m_{l+1}^{\prime}=\sharp\left\{i \mid O\left(\gamma_{j}^{(\epsilon)}-\gamma_{i}^{(\delta)}\right)=e_{i}^{\prime}\right\},
$$

where $1 \leq l \leq k$. Hence

$$
\mu_{f}\left(\gamma_{j}\right)=h-1=\sum_{i=1}^{m-1} O_{y}\left(\gamma_{j}^{(\epsilon)}-\gamma_{i}^{(\delta)}\right),
$$

and then

$$
\mu_{f}=\sum_{j=1}^{m-1} \mu_{f}\left(\gamma_{j}\right)=\sum_{j=1}^{m-1} \sum_{i=1}^{m-1} O_{y}\left(\gamma_{j}^{(\epsilon)}-\gamma_{i}^{(\delta)}\right)=\mathscr{L}\left(\mathscr{N}_{\varepsilon}, \mathscr{N}_{\delta}\right)
$$


Now, assume $d_{g r}\left(\gamma_{j}\right)=1$. In this case,

$$
\tan \theta_{\text {top }}=1, \quad \mu_{f}\left(\gamma_{j}\right)=m-1, \quad O_{y}\left(\gamma_{j}^{(\epsilon)}-\gamma_{l}^{(\delta)}\right)=1,
$$

where $1 \leq l \leq m-1$. Hence (7.4) remains true. This completes the proof.

\section{Notes}

(I) We give a proof of (1.1). (Compare [12] and [13].) First, suppose $f=c$ is a graph:

$$
f(z, w)-c=w-g(z), \quad g(z)=u(x, y)+\sqrt{-1} v(x, y),
$$

where $g(z)$ is holomorphic, $z=x+\sqrt{-1} y$. For the First Fundamental Form, we have

$$
E=G=1+\left|g^{\prime}(z)\right|^{2}=1+u_{x}^{2}+v_{x}^{2}, \quad F=0 ;
$$

and the (negative of the usual) Gaussian curvature (2], p.237) is

$$
K=\frac{1}{2 E^{3}} \cdot\left[E\left(E_{x x}+E_{y y}\right)-\left(E_{x}^{2}+E_{y}^{2}\right)\right] .
$$

Using the Cauchy-Riemann equations, we then have

$$
K=\frac{2}{E^{3}}\left[u_{x x}^{2}+v_{x x}^{2}\right]=\frac{2}{E^{3}}\left|g^{\prime \prime}(z)\right|^{2}=\frac{2\left|\Delta_{f}\right|^{2}}{\|\operatorname{Grad} f\|^{6}} .
$$

Now the general case. Near a regular point $\left(z_{0}, w_{0}\right)$ of $f(z, w)=c$, we can write

$$
f(z, w)-c=\mu(z, w)\left[\left(w-w_{0}\right)-g\left(z-z_{0}\right)\right], \quad \mu\left(z_{0}, w_{0}\right) \neq 0 \text { ( } \mu \text { a unit). }
$$

We then evaluate the derivatives at $\left(z_{0}, w_{0}\right): f_{z}=-\mu g^{\prime}, f_{w}=\mu$ and

$$
f_{z z}=-2 \mu_{z} g^{\prime}-\mu g^{\prime \prime}, f_{w w}=2 \mu_{w}, f_{z w}=-\mu_{w} g^{\prime}+\mu_{z},
$$

whence $\Delta_{f}\left(z_{0}, w_{0}\right)=\mu\left(z_{0}, w_{0}\right)^{3} g^{\prime \prime}\left(z_{0}\right)$. This completes the proof.

(II) Let us first show how to find the Newton-Puiseux coordinates of a given $\alpha_{*}$. We can apply a unitary transformation, if necessary, so that $T\left(\alpha_{*}\right)=[0: 1]$.

Take any parametrization $\beta(t)=(z(t), w(t))$ of $\alpha_{*}$. Then

$$
O_{t}(z(t))>O_{t}(w(t)), \quad \lim _{t \rightarrow 0} \frac{\|\beta(t)\|}{|w(t)|}=1 .
$$

Set $y=w(t)$. Solve $t$ as a fractional power series in $y$, then substitute it into $z(t)$ :

$$
y=w(t) \stackrel{\text { solve }}{\longrightarrow} t=\tau(y) \stackrel{\text { substitute }}{\longrightarrow} \alpha(y):=z(\tau(y)),
$$

where $\alpha(y)$, and the conjugates, are the Newton-Puiseux coordinates of $\alpha_{*}$.

Having found $\alpha(y) \in \mathbb{F}_{1}$, let us consider $\alpha_{\text {para }}(t)$ in (2.4), and write

$$
K\left(\alpha_{\text {para }}(t)\right)=a t^{N L}+\cdots, \quad a \neq 0, L \in \mathbb{Q}, \quad N:=m_{\text {puiseux }}(\alpha),
$$

where $a$ must be a positive real number since $K(z, w)$ is. We then have

$$
\lim _{t \rightarrow 0} \frac{K\left(\alpha_{\text {para }}(t)\right)}{\left\|\left(\alpha\left(t^{N}\right), t^{N}\right)\right\|^{L}}=a,
$$

since $O_{y}(\alpha(y))>1$. Now let us replace $t$ by $w(t)^{1 / N}$ in (8.2), then

$$
\lim _{t \rightarrow 0} \frac{K(\beta(t))}{\|\beta(t)\|^{L}}=\lim _{t \rightarrow 0} \frac{K\left(\alpha_{\text {para }}(t)\right)}{\left.\|\left(\alpha\left(t^{N}\right), t^{N}\right)\right) \|^{L}}=a .
$$


Thus $(a, L)$ is independent of the parametrization; $K_{*}$ is well-defined.

(III) The gradient canyons are not topological invariants. For instance, consider

$$
F(z, w)=z^{3}+w^{12}+t z^{2} w^{5}
$$

which is a topologically trivial family. At $t=0$, we have only one double polar with $d=\frac{11}{2}$, hence only one canyon. For $t \neq 0$, however, there are two disjoint canyons corresponding to the two distinct polars, both having degree $d=6$.

Gradient canyons are invariants of a stronger notion of equi-singularity, to be studied in another paper.

However, as it follows from our Theorem B, the minimality of $\mathbb{C}_{\text {enriched }}$ is a topological invariant. Indeed $f(z, w)$ has exactly $r$ distinct roots $\zeta_{i}$ in (2.10) if and only if $f$ is topologically equivalent to a homogeneous polynomial germ. This is also equivalent to $f$ having no concentration of curvature at $0 \in \mathbb{C}^{2}$ in the sense of [5] (for instance see Theorem 5.4 in [5]).

\section{REFERENCES}

1. E. Garcia Barosso and B. Teissier, Concentration multi-échelles de courbure dans des fibres de Milnor, Comment. Math. Helv. 74, No.3, 398-418 (1999).

2. M. Do Carmo, Differential Geometry of Curves and Surfaces, Prentice Hall, 1976.

3. D. Eisenbud and W. Neumann, Three-dimensional link theory and invariants of plane curve singularities, Annals of Math. Studies 110, Princeton, 1985.

4. S. Koike, T.-C. Kuo and L. Paunescu, A study of curvature using infinitesimals, Proc. Japan Acad. Ser. A, Math. Sci. 88, No. 5, 70-74 (2012).

5. S. Koike, T.-C. Kuo and L. Paunescu, Non concentration of curvature near singular points of two variable analytic functions, Several Topics on Real and Complex Singularities (JARCS4 KOBE 2011), World Scientific, 115-140 (2014).

6. T.-C. Kuo and Y. C. Lu, On analytic function germs of two complex variables, Topology 16, 299-310 (1977).

7. T.-C. Kuo and A. Parusinski, Newton polygon relative to an arc, in Real and Complex Singularities (Sao Carlos, 1998), Chapman and Hall Res. Notes Math., 412, 76-93, (2000).

8. T.-C. Kuo and L. Paunescu, Equisingularity in $R^{2}$ as Morse stability in infinitesimal calculus, (Communicated by H. Hironaka) Proc. Japan Acad. Ser. A, Math. Sci. 81, No. 6, 115-120 (2005).

9. T.-C. Kuo and L. Paunescu, Enriched Riemann Sphere, Morse Stability and Equi-singularity in $\mathcal{O}_{2}$, Jour. London Math. Soc. 85, 382-408 (2012).

10. R. Langevin, Courbure et singularités complexes, Comment. Math. Helv. 54, 6-16 (1979).

11. J. Milnor, Singular Points of Complex Hypersurfaces, Annals of Math. Studies 61, Princeton, 1968.

12. L. Ness, Curvature on algebraic plane curves.I, Compositio Mathematica, tome 35, no. 1 (1977), 57-63.

13. S. Nishimura and M. Hashiguchi, On the Gaussian Curvature of the Indicatrix of a Lagrange space, Rep. Fac. Sci. Kagoshima Univ., (Math. Phys. \& Chem.), No. 24, 33-41 (1991).

14. D. Siersma and M. Tibar, The Gauss-Bonnet defect of complex affine hypersurfaces, Bull. Sci. Math. 130, No. 2, 110-122 (2006).

15. J. E. Reeve, A summary of results in the topological classification of plane algebroid singularities, Rendiconti Sem. Mat. Torino. 51, 159-187 (1954-55).

16. Robert J. Walker, Algebraic Curves, Dover, 1962.

17. C. T. C. Wall, Singular Points of Plane Curves, London Math. Soc. Student Text 63, Cambridge, 2004. 
A'CAMPO CURVATURE BUMPS AND THE DIRAC PHENOMENON NEAR A SINGULAR POINT 35

Department of Mathematics, Hyogo University of Teacher Educhtion, Hyogo, Japan; School of Mathematics and Statistics, University of Sydney, Sydney, NSW, 2006, Australia.

E-mail address: koike@hyogo-u.ac.jp; tzeechar@gmail.com; laurent@maths.usyd.edu.au 